\title{
Estimating V02max Using a Personalized Step Test
}

Catherine Webb

Brigham Young University - Provo

Follow this and additional works at: https://scholarsarchive.byu.edu/etd

Part of the Exercise Science Commons

\section{BYU ScholarsArchive Citation}

Webb, Catherine, "Estimating V02max Using a Personalized Step Test" (2012). Theses and Dissertations. 3526.

https://scholarsarchive.byu.edu/etd/3526

This Thesis is brought to you for free and open access by BYU ScholarsArchive. It has been accepted for inclusion in Theses and Dissertations by an authorized administrator of BYU ScholarsArchive. For more information, please contact scholarsarchive@byu.edu, ellen_amatangelo@byu.edu. 
Estimating $\mathrm{VO}_{2} \max$ Using a Personalized Step Test

\author{
Catherine Webb \\ A thesis submitted to the faculty of \\ Brigham Young University \\ in partial fulfillment of the requirements for the degree of
}

Master of Science

Pat Vehrs, Chair

James George

Ron Hager

Department of Exercise Sciences

Brigham Young University

June 2012

Copyright (C) 2012 Catherine Webb

All Rights Reserved 


\author{
ABSTRACT \\ Estimating $\mathrm{VO}_{2}$ max Using a Personalized Step Test \\ Catherine Webb \\ Department of Exercise Sciences, BYU \\ Master of Science
}

The purpose of this study was to develop a personalized step test and a valid regression model that used non-exercise data and data collected during the step test to estimate $\mathrm{VO}_{2} \mathrm{max}$ in males and females 18 to 30 years of age. All participants $(\mathrm{N}=80)$ successfully completed a step test with the starting step rate and step height being determined by the self-reported perceived functional ability (PFA) score and participant's height, respectively. All participants completed a maximal graded exercise test (GXT) to measure $\mathrm{VO}_{2} \max$. Multiple linear regression analysis yielded the following equation $\left(R=0.90, S E E=3.43 \mathrm{~mL} \cong \mathrm{kg}^{-1} \cong \mathrm{min}^{-1}\right): 45.938+9.253(\mathrm{G})-$ $0.140(\mathrm{KG})+0.670(\mathrm{PFA})+0.429(\mathrm{FSR})-0.149(45 \mathrm{sRH})$ to predict $\mathrm{VO}_{2} \mathrm{max}\left(\mathrm{mL} \cong \mathrm{kg}^{-1} \cong \mathrm{min}^{-1}\right)$ where: $\mathrm{G}$ is gender $(0=$ female; $1=$ male $), \mathrm{KG}$ is body mass in $\mathrm{kg}$, PFA is the sum of the two PFA questions, FSR is the final step rate (step-ups/min), and $45 \mathrm{sRHR}$ is the recovery heart rate 45 seconds following the conclusion of the step test. Each independent variable was significant $(p<$ $0.05)$ in predicting $\mathrm{VO}_{2} \max$ and the resulting regression equation accounted for roughly $83 \%$ $\left(R^{2}=0.8281\right)$ of the shared variance of measured $\mathrm{VO}_{2}$ max. Based on the standardized $\beta$-weights, gender (0.606) explained the largest proportion of variance in $\mathrm{VO}_{2}$ max values followed by PFA (0.315), body mass (-0.256), FSR (-0.248), and the 45sRHR (-0.238). The cross validation statistics $\left(R_{P R E S S}=0.88, S E E_{\text {PRESS }}=3.57 \mathrm{~mL} \cong \mathrm{kg}^{-1} \cong \mathrm{min}^{-1}\right)$ show minimal shrinkage in the accuracy of the regression model. This study presents a relatively accurate model to predict $\mathrm{VO}_{2}$ max from a submaximal step test that is convenient, easy to administer, and individualized.

Keywords: Step test, $\mathrm{VO}_{2} \mathrm{max}$, cardiorespiratory fitness, PFA 


\section{ACKNOWLEDGEMENTS}

To Pat Vehrs, for making this possible with his patience, effort, encouragement, advice and countless hours of work.

To Patrick Webb for supporting me, giving me hope, sacrificing his time, always putting priorities first, tending Logan, and never giving up on me.

To Amy Jo Webb for tending Logan, and caring about my project.

To Brent Webb for being interested in my work, supporting me, and attending my meetings.

To Logan for being such a good kid and letting me work on this the first years of his life.

To my mother for installing in me a love of exercise and healthy foods from an early age.

To my father for being a great example of a university professor and parent.

To secretaries Sandy Alger, Sharron Collier and Maggie Shibla for their support.

To Jessica Crandall and Jeanine Wagner for volunteering with data collection.

To Mary Lou and Ira Fulton for funding this project. 


\section{Table of Contents}

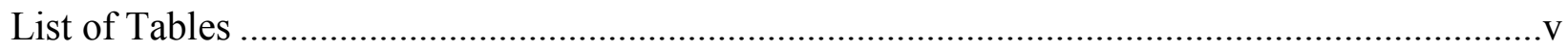

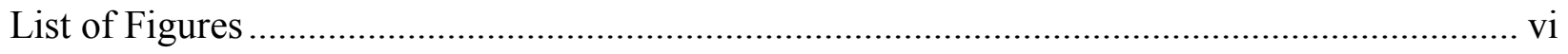

Personalization of the Step Test

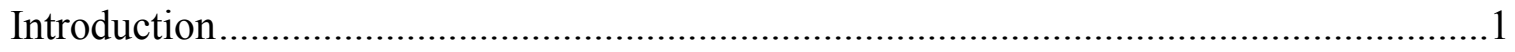

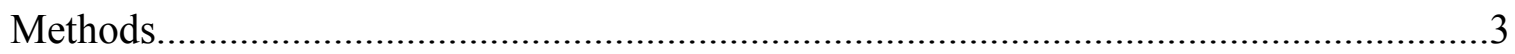

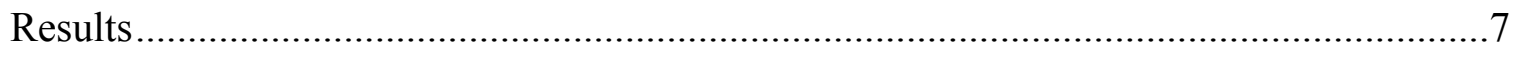

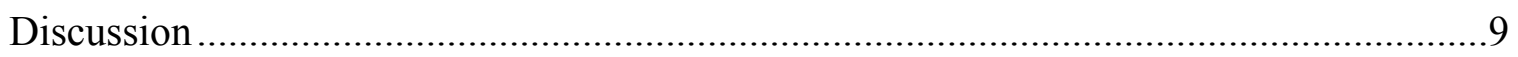

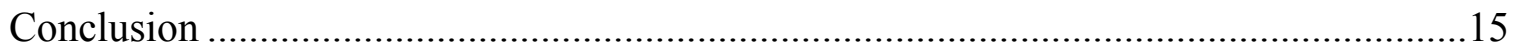

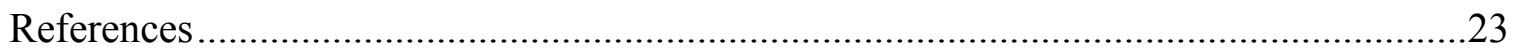

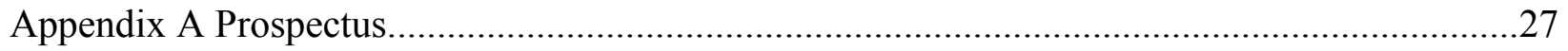

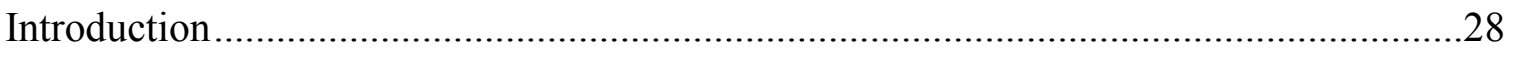

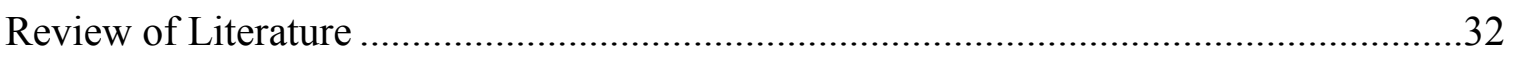

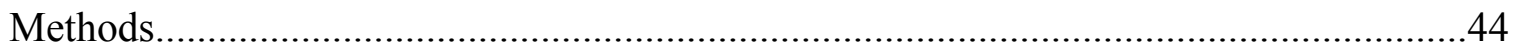

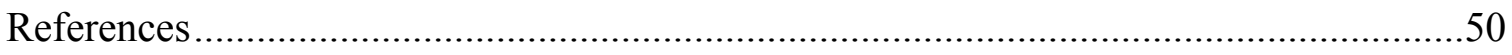

Appendix A-1 Perceived Functional Ability (PFA) Questions ..............................................55

Appendix A-2 Physical Activity Rating (PA-R) ..........................................................56

Appendix A-3 Physical Activity Readiness Questionnaire (PAR-Q) ......................................57

Appendix A-4 Borg Rate of Perceived Exertion Scale .......................................................58 


\section{List of Tables}

\section{Tables}

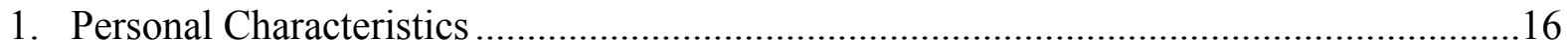

2. Maximal Exercise Test Results............................................................................... 17

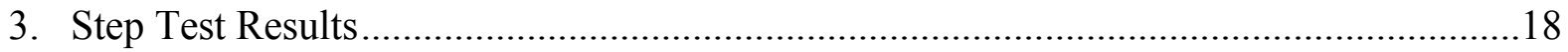

4. Regression Model to Predict $\mathrm{VO}_{2}$ max from Step Test Data........................................19 


\section{List of Figures}

\section{Figures}

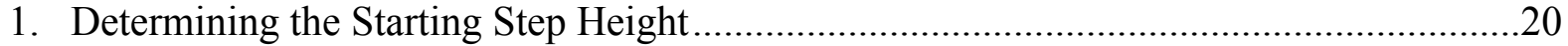

2. Determining the Starting Step Rate (Steps/min) .....................................................21

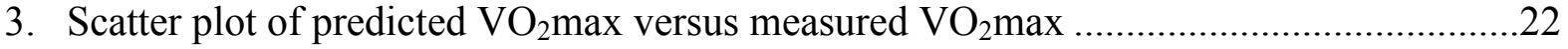




\section{Introduction}

Cardiorespiratory fitness (CRF) is one of the components of health-related physical fitness. Cardiorespiratory fitness is defined as the amount of oxygen the body can utilize during strenuous aerobic exercise for an extended time (Brooks, Fahey \& Baldwin 2005). Higher levels of CRF are related to a decreased risk for cardiovascular disease, coronary heart disease, stroke, obesity, and type 2 diabetes and positive changes in overall wellbeing.

Prior to starting an exercise program, an assessment of CRF can be useful for designing a safe and effective regimen. Information about CRF obtained from a baseline assessment can also be educational, motivational and useful for identifying progress and improvement. The most accurate measure of CRF is the maximum amount of oxygen a person is able to consume during strenuous exercise, also known as maximum oxygen uptake, or $\mathrm{VO}_{2} \mathrm{max}$ (Santo \& Golding, 2003).

The standard test for measuring CRF involves performing a maximal graded exercise test (GXT) and the measurement of $\mathrm{VO}_{2}$ max using a metabolic cart. Maximal GXTs are typically not well tolerated by the overweight, less physically fit, or elderly due to the demanding, maximal effort required by the participant (Santo \& Golding, 2003, Watkins, 1984). It is also generally reserved for clinical, laboratory, or research settings because it is time consuming, costly and requires the use of trained personnel. Due to the limitations associated with the direct measurement of $\mathrm{VO}_{2} \mathrm{max}$, a variety of less demanding submaximal exercise tests have been developed to predict CRF (Draper \& Jones 1990; Leger, Mercier, Gadoury, \& Lambert, 1988; Francis \& Brasher, 1992; George, Vehrs, Allsen, Fellingham, \& Fisher,1993). Step tests have long been used to predict $\mathrm{VO}_{2} \max$ (Brouha, Fradd, \& Savage 1944; Rhyming 1954). During the step test, a participant usually steps up and down on a bench of a specific height for 3-5 minutes 
at a specific cadence, or step rate. Heart rate (HR) during the recovery period is typically used to estimate CRF. Although the step test is a simple, quick, and easily administered test to predict $\mathrm{VO}_{2} \mathrm{max}$ individually or in groups, we suspect that the step test is not commonly used today because of poor predictive accuracy and because the required step height or step rate are not matched to the participant's fitness level (Siconolfi, Garber, Lasater \& Carleton et al., 1985; Brouha, Fradd \& Savage, 1944). Stepping may also not be the testing mode of choice due to the increasing popularity of treadmills and cycle ergometers. Nevertheless, stepping is a popular form of exercise in group exercise classes and currently available exercise steps allow for easy adjustment of step height to accommodate differences in fitness level or stature. A step test that personalizes step height and step rate may improve the test's validity and therefore the ability to accurately estimate $\mathrm{VO}_{2}$ max.

Non-exercise data can also be used to predict CRF. Questionnaires have been used as a tool for participants to self-report their current level of physical activity (PA-R; Jackson, Blair, Mahar, Wier, Rossand \& Stuteville, 1990) or perceived functional ability (PFA; George, Stone \& Burkett, 1997) to exercise for one or three miles. The PA-R and PFA scores have been used to develop non-exercise predictions of CRF or provide an additional independent variable in prediction equations that estimate $\mathrm{VO}_{2} \max$ from data gathered during a submaximal exercise test. In 2009 George, Paul, Hyde, Bradshaw, Vehrs, \& Hager et al., generated a regression equation using the PFA, a modified PA-R scale, and other non-exercise data (age, gender, BMI) as predictor variables along with exercise data (HR and work rate) from a submaximal treadmill test to predict $\mathrm{VO}_{2}$ max. The accuracy of the prediction equation that included both exercise and non-exercise variables exceeded $\left(S E E=3.09 \mathrm{~mL} \cdot \mathrm{kg}^{-1} \cdot \mathrm{min}^{-1} ; R=0.94\right)$ that of previous prediction equations using either exercise or non-exercise data. 
A step test in which the starting step height and step rate are determined by the selfreported physical activity levels or PFA could effectively personalize the step test. In addition, use of non-exercise variables such as the PFA and PA-R in conjunction with exercise data (e.g., HR, step height, and step rate) could increase the accuracy of a regression equation to predict $\mathrm{VO}_{2} \mathrm{max}$. The efficacy of using physical activity data to personalize a step test or using nonexercise data as predictor variables in a regression model to predict $\mathrm{VO}_{2}$ max from a step test has not been studied. Therefore, the purpose of this study was to develop a valid regression model that used non-exercise data and data collected during a personalized step test to estimate $\mathrm{VO}_{2} \mathrm{max}$ in males and females 18 to 30 years of age.

\section{Methods}

\section{Participants and Procedures}

The participants in this study included 51 males and 47 females, 18-29 years of age. Each participant completed a pre-participation questionnaire to screen for conditions that increase the risk of cardiovascular, pulmonary, or metabolic events during exercise testing. All participants were classified as "low risk" according to the guidelines of the American College of Sports Medicine (ACSM, 2010). All methods and procedures of this study were reviewed and approved by the Institutional Review Board for Human Subjects and all participants provided written informed consent prior to participation in this study.

Each participant completed the PFA questionnaire and the PA-R questionnaire (George et al., 1997). The participant's PFA score was calculated as the sum of the two PFA questions. Each participant's mass $(\mathrm{kg})$ and height $(\mathrm{cm})$ were measured and recorded to the nearest onetenth of a kilogram and to the nearest one-half centimeter, respectively, using a digital weight 
scale (Ohaus Model CD-33, Ohaus Corporation, Pine Brook NJ, USA) and a calibrated wall scale.

Each participant performed a maximal graded exercise test (GXT) on the treadmill and a submaximal step test. During the submaximal step test and the maximal GXT, heart rate was monitored using a radiotelemetry heart-rate monitor (Polar Electro OY, Hong Kong) and rating of perceived exertion (RPE) was monitored during each test using the Borg 15-point scale (Noble, Borg, \& Jacobs, 1983). Participants were instructed to (a) wear comfortable clothes and shoes appropriate for exercise, (b) drink plenty of fluids over the 24-hour period prior to exercise testing to ensure normal hydration, (c) refrain from eating food other than water, and from using tobacco, alcohol, and caffeine for two to three hours prior to exercise testing, and (d) avoid exercise or strenuous physical activity the day of the testing. Step Test

Each participant completed a multistage step test that was developed through pilot experimentation. The step test was performed on The Step (The Step, Inc., Marietta, GA) with three or more sets of risers to set the step height at 10 to 16 inches. The starting step height (Figure 1) was determined by using the Francis and Culpepper equation ( $0.19 \mathrm{x}$ participant height (cm); 1989) which allows the participant to have an ideal hip angle of 73.3 degrees. To further personalize the step test, the participant began the step test at stepping rates of 10, 15, 20, or $25 \mathrm{steps} / \mathrm{min}$ (Stage I, II, III, or IV, respectively) depending on the their PFA score (Figure 2).

The participant's resting HR was recorded prior to beginning exercise testing and $75 \%$ of their age-predicted maximal HR was calculated using the formula, (207- $\left(0.7^{*}\right.$ age $\left.)\right)$; Tanaka, Monahan \& Seals, 2001). Each was familiarized with the four-step sequence of up/up, down/down before the exercise test. Participants were instructed to keep their knees and back 
straight at the top of each step. A metronome was used to help participants maintain the required cadence (steps/min) during the pre-test familiarization and during the exercise test. Following familiarization, participants began the step test at their personalized step height (Figure 1) and step rate (Figure 2). The step test developed in this study incorporated the Chester Step Test's two-minute stage protocol (Buckley, Sim, Eston, Hession, \& Fox, 2004). After two minutes stepping rate was increased by 5 steps/min and continued in like manner every two minutes until the HR during the last $30 \mathrm{~s}$ of the stage was equal to or greater than $75 \%$ age-predicted maximal HR. Heart-rate and RPE were recorded near the end of each two minute stage. When the HR reached $75 \%$ of age-predicted maximal HR, the participant finished the current stage and the test was terminated. Immediately following the completion of the step-test, participants assumed a seated position and HR was recorded immediately post-exercise and every $15 \mathrm{~s}$ thereafter for one minute. The HR and RPE from the last step test stage completed, post-exercise HRs, step height, and final stepping rate were used as independent variables in the statistical analysis to predict $\mathrm{VO}_{2} \max$.

If participants began the step test at an appropriate step height and step rate, it was expected that they would complete two or more stages of the step test. If a participant reached $75 \%$ of their age-predicted maximal HR in their first two-minute stage, the test was terminated and they repeated the test starting at a lower height after resting for sufficient time for HR to return to pre-exercise testing resting levels.

Following the completion of the step test, participants had a 10 to 15 minute active and resting recovery before completing a maximal GXT on a motor-driven treadmill (Model TMX425C, Full Vision, Inc., Newton, KS). Metabolic and ventilatory responses to exercise were measured using a Truemax 2400 metabolic cart (Consentious Technologies, Sandy, UT). 
Prior to each maximal GXT, the flow meter was calibrated at five different flow rates using a 3-L syringe and the oxygen and carbon dioxide analyzers were calibrated using room air and a medical grade calibration gas of known concentrations. The metabolic cart was programmed to display and print metabolic and ventilatory data every 15 seconds. Participants were fitted with a mouthpiece, one-way breathing valve, and a nose clip to aid in the measurement of expired air and gases.

Each participant completed a maximal GXT similar to a protocol previously described (George et al., 2009). During the first minute on the treadmill, participants were instructed to walk at a comfortable pace. From minute one to minute two, participants were instructed to choose a treadmill jogging speed that they could comfortably maintain for 25 to 30 minutes. Treadmill speed remained constant during the remaining stages of the exercise test and treadmill grade was increased $1.5 \%$ starting after the third minute, and every minute thereafter until the subject voluntarily terminated the test due to fatigue, despite verbal encouragement. After terminating the test, participants walked at a self-selected speed at level grade for any desired amount of time to cool down.

The participant's effort during the maximal GXT was considered maximal if physical signs representative of exhaustion were obvious and at least two of the following four criteria were met: (a) maximal respiratory exchange ratio (RER) greater than or equal to 1.10 , (b) a maximal HR that was no less than 15 beats below age-predicted maximal HR, (c) a RPE of 19 or 20 on Borg's RPE scale, and (d) a leveling off of $\mathrm{VO}_{2}$. Maximal HR was defined as the highest single $\mathrm{HR}$ value during the GXT and $\mathrm{VO}_{2}$ max was defined as the highest 30 -s average $\mathrm{VO}_{2}$ value during the final minutes of the exercise test. 
Statistics

Of the original 98 participants, data on 18 participants (13 male and 5 female) were discarded because they had cardiorespiratory fitness levels that were unusually high for college age males and females $\left(\mathrm{VO}_{2} \max >60 \mathrm{~mL} \cdot \mathrm{kg}^{-1} \cdot \mathrm{min}^{-1} ; \mathrm{n}=7\right)$, inability to record heart rate accurately $(n=2)$, or outlying data $(n=9)$. Statistical software (SAS) was used to generate a $\mathrm{VO}_{2} \mathrm{max}$ regression model using age, gender, body weight, body mass index (BMI), step height, final step rate, ending HR, recovery HRs (15s, 30s, 45s, and 60s), PFA score, PA-R score, and any reasonable two-way interactions of the remaining 80 participants. The relative accuracy of the model was evaluated based on the computed correlation coefficient and the standard error of estimate (SEE). Predicted residual sum of squares (PRESS) statistics (Holiday, Ballard, \& McKeown, 1995) was used to estimate the degree of shrinkage one could expect when the $\mathrm{VO}_{2}$ max prediction equation is applied to a similar but independent sample. An alpha level of $\mathrm{p}$ $<0.05$ was maintained in all analyses.

\section{Results}

The descriptive data of the 38 male and 42 female participants who completed this study are included in Table 1. On average $( \pm \mathrm{SD})$, males $(23.7 \pm 2.2$ years, $1.79 \pm 0.07 \mathrm{~m}, 78.1 \pm 13.1$ $\mathrm{kg})$ were slightly older, taller, and heavier than their female counterparts $(21.4 \pm 2.6$ years, 1.65 $\pm 0.06 \mathrm{~m}, 66.3 \pm 12.4 \mathrm{~kg})$, respectively. Participants' PFA and PA-R scores ranged from 10 to 26 and 1 to 10 , respectively. Males and females had similar physical activity levels as noted by their self-reported PAR and PFA values.

Graded maximal exercise test results are included in Table 2. All of the participants included in the data analysis achieved a valid $\mathrm{VO}_{2}$ max during the maximal GXT. The overall average $( \pm \mathrm{SD}) \mathrm{VO}_{2}$ max was $47.6 \pm 7.7 \mathrm{~mL} \cdot \mathrm{kg}^{-1} \cdot \mathrm{min}^{-1}$. The corresponding RER $(1.12 \pm 0.03)$, 
HR $(191.7 \pm 9.6 \mathrm{bpm} ; 97.1 \pm 4.8$ percent of age predicted maximal HR), and RPE $(19.2 \pm 0.8)$ responses reflect a maximal effort during the exercise test. As expected, the average cardiovascular fitness level (i.e., $\left.\mathrm{VO}_{2} \mathrm{max}\right)$ was greater in the male $\left(52.1 \pm 4.9 \mathrm{~mL} \cdot \mathrm{kg}^{-1} \cdot \mathrm{min}^{-1}\right)$ participants than in the female $\left(43.6 \pm 5.9 \mathrm{~mL} \cdot \mathrm{kg}^{-1} \cdot \mathrm{min}^{-1}\right)$ participants. The measured $\mathrm{VO}_{2} \mathrm{max}$ values are typical of college aged adults.

The results of the step test are included in Table 3. Compared to their male counterparts, females tended to begin the step test at lower-level stages. For example, one female began the step stest at Stage 1 and 10 began the step test at Stage 2 whereas none of the males began the step test at Stage 1 and only 3 males began the step test at Stage 2. Likewise, more males completed Stage 5 and Stage 6 than did females. This can be attributed to the higher cardiovascular fitness level in males, compared to females. Seven participants reached their target HR (i.e., 75\% of age predicted maximal HR) during their first stage. In all of these participants, this was attributed to self-reporting a PFA score that was too high, which resulted in starting the step test at a step rate that was too challenging. These participants were allowed to rest until their HR returned to near pre-test resting values and then started the step test again at a lower step rate. Three of these participants reached their target heart rate during the first stage of the step test even when starting at a lower level.

The multiple linear regression analysis yielded the following equation $(R=0.90, S E E=$ $3.43 \mathrm{~mL} \cdot \mathrm{kg}^{-1} \cdot \mathrm{min}^{-1}$, Table 4) to predict $\mathrm{VO}_{2} \max \left(\mathrm{mL} \cdot \mathrm{kg}^{-1} \cdot \mathrm{min}^{-1}\right)$ :

$$
45.938+9.253(\mathrm{G})-0.140(\mathrm{WT})+0.670(\mathrm{PFA})+0.429(\mathrm{FSR})-0.149(45 \mathrm{sRHR})
$$

where: $\mathrm{G}$ is gender $(0=$ female; $1=$ male $)$, WT is body weight in $\mathrm{kg}$, PFA is the PFA score, FSR is the final step rate (step-ups/min ), and $45 \mathrm{sRHR}$ is the recovery heart rate 45 seconds following the termination of the step test. The cross validation statistics $\left(R_{P R E S S}=0.88, S E E_{P R E S S}=3.57\right.$ 
$\mathrm{mL} \cdot \mathrm{kg}^{-1} \cdot \mathrm{min}^{-1}$; Table 4) show minimal shrinkage in the accuracy of the regression model. As expected, body mass $(-0.110)$ and the $45 \mathrm{sRHR}(-0.280)$ were negatively correlated to $\mathrm{VO}_{2} \max$. Each of the independent variables was significant $(p<0.05)$ in predicting $\mathrm{VO}_{2} \mathrm{max}$ and the resulting regression equation accounted for roughly $83 \%\left(R^{2}=0.8281\right)$ of the shared variance of measured $\mathrm{VO}_{2}$ max. Based on the standardized $\beta$-weights (Table 4), gender (0.606) explained the largest proportion of variance in $\mathrm{VO}_{2}$ max values followed by PFA (0.315), body mass (-0.256), FSR (-0.248), and the 45sRHR (-0.238). Figure 3 is a scatter plot of the predicted versus measured $\mathrm{VO}_{2}$ max values for the male and female participants in this study.

\section{Discussion}

This study is the first to combine exercise HR responses to a step test and non-exercise data to predict CRF. The two most compelling findings of this study are that self-reported PFA can be used to personalize a step test and is a significant independent variable in the accurate prediction of CRF in college age males and females.

Previously developed step tests are often not well suited to the participant because step height or step rate are not matched to the individual's fitness level (Watkins, 1984; Keen \& Sloan, 1957). Performance on a single-stage step test that uses a fixed step height and step rate may be limited by muscular fatigue (Watkins, 1984). The step height or step rate of some step tests have been modified based on gender or height of the participant. Rhyming (1954) used a bench height of $33 \mathrm{~cm}$ for females and $40 \mathrm{~cm}$ for males and a constant stepping rate of $22.5 \mathrm{step}$ ups/min. The step test developed by Culpepper and Francis (1987) used $0.189 \mathrm{x}$ height (cm) of male and $0.192 \times$ height $(\mathrm{cm})$ of female participants to determine the step test height. The Canadian Home Fitness Test (Bailey, Shephard \& Mirwald, 1976) used a step rate based on age of the participant and a six-step sequence. The Siconolfi Step Test (1985) used a set step height 
of $25.4 \mathrm{~cm}$ and incremental step rates $(17,26$ and 34 step-ups/min) for three stages. Petrella, Koval, Cunningham \& Paterson (2001) allowed elderly participants to select their own step rate. Although the Chester Step Test (Buckley et al., 2004) determines step height based on age and activity level, the measure of activity level (Sykes, 1998) is not readily available and its use has not been reported elsewhere.

No other step tests have personalized the step test based on the physical activity level or perceived fitness level of the participant. This study developed a personalized multistage step test in two ways: we adjusted the step height for each participant based on the equation reported by Francis and Culpepper (1989); and we determined the beginning step rate based on the participant's self-reported PFA. Using a personalized step height and beginning stage for the step test eliminates the need to personalize the step test based on gender or age. The primary benefit of using PFA to determine the starting stage of the step test is that it restrains the step test to a reasonable duration regardless of fitness level. Our objective in using a personalized step height and starting stage was to have a participant complete the step test in two to three stages. Although the step test developed in this study allows for a participant to begin the step test using a step height of 10 or 16 inches (Figure 1), all participants in this study began the step test at either 12 inches $(\approx 30 \mathrm{~cm} ; \mathrm{N}=44)$ or 14 inches $(\approx 35 \mathrm{~cm} ; \mathrm{N}=36)$. One participant began the step test at Stage 1 (10 steps/min), 11 participants began at Stage 2 (15 steps/min), 50 participants began at Stage 3 (20 steps $/ \mathrm{min})$ and 18 participants began at Stage 4 ( $25 \mathrm{steps} / \mathrm{min})$. Three participants completed the step test in one stage, 43 participants completed two stages, 31 participants completed three stages, and 3 participants completed the step test in four stages. The participants in this study completed the step test in an average time of $4.8 \mathrm{~min} \pm 1.3 \mathrm{~min}$. As 77 
of the 80 participants (96\%) in this study completed the step test within three 2 -minute stages it is reasonable to expect that once started, the step test would take less than 6 minutes to complete.

Numerous regression models that predict CRF based on data gathered during a submaximal or maximal exercise test are available. The relationship between self-reported physical activity and CRF has previously been reported (Siconolfi, Lasater, Snow \& Carleton, 1985) and regression models that include only non-exercise data (e.g., age, gender, body weight, body mass index, and self-reported physical activity) appeared in the 1990's (Jackson et al., 1990; Ainsworth, Richardson, Jacobs, \& Leon,1992; Heil, Freedson, Ahlquist, Price, \& Rippe, 1995; George, et al, 1997). More recent studies have combined exercise data and non-exercise data to predict CRF (George et al., 2009; Nielson, George, Vehrs, Hager, \& Webb, 2010). No other studies have combined exercise test data and self-reported physical activity or fitness levels to predict CRF from a step test. Previously developed step tests include variables such as recovery heart rate, exercise heart rate, test duration, age, gender, and body weight to predict $\mathrm{VO}_{2} \max$ (McArdle, Katch, Pechard, Jacobson \& Ruck, 1972; Shephard, 1980; Siconolfi et al., $1985)$.

Previous studies have reported PFA to be a significant predictor of CRF using cycling (Nielsen et al, 2010), treadmill walking, jogging, and running (George et al., 2009), and only non-exercise estimates of CRF (George et al, 1997; Bradshaw, George, Hyde, LaMonte, Vehrs, \& Hager, et al., 2005). The results of this study are similar to these previous studies. For example, the first study to use PFA as an independent variable to predict CRF (George et al.,1997) reported an $R=0.85$ and a $S E E=3.44 \mathrm{~mL} \cdot \mathrm{kg}^{-1} \cdot \mathrm{min}^{-1}$. The rank order of the beta weights of the independent variables included in the regression equation to predict CRF in this study indicate that there is a greater contribution from the PFA score $(0.315)$ than body mass $(\mathrm{kg}$; - 
0.256), final step rate (steps/min; 0.248), and recovery HR (bpm; -0.238). This concurs with previous studies that ranked the beta weight of PFA higher than all other variables (Bradshaw et.al., 2005), third after gender and body mass (Nielson et al., 2010), and fourth after treadmill speed, age, and gender (George et al., 2009). When the PFA variable was removed from the full model, the $R$ value decreased from 0.91 to 0.87 , the variance explained by the regression model decreased by $8.6 \%$ (from $82.8 \%$ to $75.7 \%$ ), and the SEE increased by $11.9 \%$ (from $3.43 \mathrm{~mL} \cdot \mathrm{kg}^{-}$ ${ }^{1} \cdot \mathrm{min}^{-1}$ to $3.84 \mathrm{~mL} \cdot \mathrm{kg}^{-1} \cdot \mathrm{min}^{-1}$ ). This suggests that participants' perception of their ability to comfortably walk, jog, or run one and three mile distances accounts for a significant portion of the variance beyond that accounted for by other independent variables (i.e., step rate, HR). Inclusion of PFA in prediction models provides a better explanation of a person's CRF than what can be explained by submaximal exercise test data alone.

Previous studies have included PA-R in regression models to predict CRF (Bradshaw et al, 2005; George et al, 1997, Jackson et al, 1990). In the present study, although both PA-R and PFA values were evaluated as potential independent variables, only PFA was statistically significant (Table 4). The PA-R variable only entered into the model if the PFA score was excluded. One possible explanation for this is that the range of self-reported PA-R scores (1 to 10) was narrower than the range of self-reported PFA scores (10 to 26).

Other independent variables important to the prediction of CRF include age, gender, and body mass. Although age is generally inversely related to CRF, because this study involved a relatively homogenous sample of college students ( $18 ¥ 29$ years of age), age was not statistically significant in the prediction of CRF. Consistent with other research (George et al., 2009; Nielson, et al., 2010) the results of this study found gender to be a significant predictor of CRF. The male participants $\left(52.1 \pm 6.5 \mathrm{~mL} \cdot \mathrm{kg}^{-1} \cdot \mathrm{min}^{-1}\right)$ in this study had average $\mathrm{VO}_{2}$ max values that were $19.5 \%$ 
greater than that of their female $\left(43.6 \pm 6.4 \mathrm{~mL} \cdot \mathrm{kg}^{-1} \cdot \mathrm{min}^{-1}\right)$ counterparts. The beta weight for gender (0.606) was the highest of all the variables included in the regression model (Table 4), indicating the importance of its contribution to the prediction of CRF.

Traditional methods of cross validating a regression equation involve partitioning the data into validation and cross validation groups. PRESS-related statistics are an alternative crossvalidation technique for regression models built from small data sets (Holiday, 1995). The advantage of using PRESS-related statistics is that the entire data set can be used to build the regression model. In this study, data from all 80 subjects were used to build the regression model (Table 4) and the PRESS statistics estimate the degree of shrinkage that could be expected when the regression model is applied to similar but independent data sets. Thus, use of the regression model developed in this study on independent cross-validation samples should yield estimates of $\mathrm{VO}_{2}$ max that approximates the PRESS statistics presented in Table $4\left(R=0.88, S E E=3.57 \mathrm{~mL} \cdot \mathrm{kg}^{-}\right.$ $\left.{ }^{1} \cdot \min ^{-1}\right)$

The step test developed in this study is a multistage step test with each stage representing an increment in physical work. The incremental nature of the step test presents a progressive challenge to the cardiorespiratory system with each participant terminating the step test at the same relative end-point (i.e., 75\% of age predicted maximal HR) regardless of age, gender, or fitness level. In this study the average HR during the final stage of the step test was $152 \mathrm{bpm}$ (range $142 \mathrm{bpm}$ to $170 \mathrm{bpm} ; 79.5 \pm 4.6 \%$ age predicted maximal HR). Due to individual difference in fitness level, single stage step tests that use fixed step heights and step rates do not present the same relative cardiorespiratory challenge to each participant. Multistage step tests that have as their end point a target HR (percent of age predicted maximal HR) provide a similar relative challenge to each participant. The primary disadvantage of this multistage step test is 
that it is not conducive to a group setting (e.g., a physical education class) in which participants are using a different step height and stepping at a different cadence. Nevertheless, an individualized multistage step test is applicable to a variety of situations in which the cardiorespiratory fitness of an individual is being assessed.

The HR response during the recovery period immediately following exercise can be used as an indicator of an individual's CRF level. Generally, the faster one's HR returns to a resting HR following exercise, the higher the fitness level. The usefulness of recovery HR in predicting CRF depends on when it is recorded following exercise (Watkins, 1984). Watkins and Ewing (1984) reported that compared to pulse counts measured at 1 minute and 2 minutes into recovery, pulse counts measured within the first 20 s of recovery had the highest reliability coefficient $(R=$ 0.94). Based on these findings, a pulse count taken during the first 30 s of recovery has been recommended (Watkins, 1984). The findings of McArdle et al. (1972) concur with this recommendation. McArdle et al. reported that a 15s HR recorded between 5-20s into recovery was most highly correlated $(R=-0.76)$ to $\mathrm{VO}_{2}$ max. In our study the recovery HR recorded at $45 \mathrm{~s}$ was most highly correlated $(R=-0.280)$ to $\mathrm{VO}_{2}$ max compared to the HR recorded during the final stage $(R=-0.164), 15 \mathrm{~s}$ into recovery $(R=-0.240), 30$ s into recovery $(R=-0.250)$, and 60 s into recovery $(R=-0.263)$. The discrepancy between the correlation reported in this study $(R=-0.280)$ and that reported by McArdle et al. $(R=-0.760)$ may be due to the method of measuring HR. McArdle et al. measured HR during the recovery period of the step test by palpation. In this study, we measured HR using an electronic chest-strap type HR monitor as opposed to palpating a radial or carotid pulse. The availability of HR monitors allows for the rapid and accurate measurement of HR at specific points in time instead of over a 15, 30, or 60s time interval. 
Further research is warranted to validate this step test in other samples of participants with various levels of CRF. It is foreseeable that the model developed in this study may require an additional age variable as research cross validates the regression equation in adolescents and men and women beyond college age years.

\section{Conclusion}

The step test and the accompanying regression model developed in this study provide an accurate estimate of $\mathrm{VO}_{2} \max$ using exercise and non-exercise data. The results from this study demonstrate the ability of PFA to personalize the starting step rate of the multistage step test. In addition, the PFA data is easy to collect and improves the prediction of CRF in college age men and women. Although other modes of exercise (e.g., treadmills, elipticals, cycling) are popular, stepping is also a popular form of exercise in group exercise classes. The protocol developed in this study allows a participant to perform a step test using an individualized step height and a step rate appropriate for their fitness level. The step test is accurate, safe, and cost and time effective. The validity of the regression model developed in this study makes this step test an excellent choice for use in a variety of school, employment, and fitness settings in which individual assessments of $\mathrm{CRF}$ are made. Further research is warranted to cross validate this step test in similar but independent samples as well as evaluate the influence of age in younger and older samples. Research can also evaluate the accuracy of this step test when it is selfadministered. 
Table 1. Personal Characteristics

\begin{tabular}{|c|c|c|c|c|c|c|}
\hline \multirow[b]{2}{*}{ Age (years) * } & \multicolumn{2}{|c|}{$\begin{array}{l}\text { Male } \\
(n=38)\end{array}$} & \multicolumn{2}{|c|}{$\begin{array}{l}\text { Female } \\
(n=42)\end{array}$} & \multicolumn{2}{|c|}{$\begin{array}{l}\text { Combined } \\
(n=80)\end{array}$} \\
\hline & $23.7 \pm$ & $\pm \quad 2.2$ & $21.4 \pm$ & 2.6 & $22.5 \pm$ & 2.7 \\
\hline Height $(m) *$ & $1.79 \pm$ & $\pm \quad 0.07$ & $1.65 \pm$ & 0.06 & $1.72 \pm$ & 0.10 \\
\hline Weight $(\mathrm{kg}) *$ & $78.1 \pm$ & \pm 13.1 & $66.3 \pm$ & 12.4 & $71.9 \pm$ & 13.9 \\
\hline BMI $\left(\mathrm{kg} / \mathrm{m}^{2}\right)$ & $24.1 \pm$ & $\pm \quad 3.4$ & $24.2 \pm$ & 4.0 & $24.1 \pm$ & 3.7 \\
\hline PA-R & $5.7 \pm$ & $\pm \quad 1.9$ & $6.1 \pm$ & 2.0 & $5.9 \pm$ & 2.0 \\
\hline PFA score & $18.7 \pm$ & $\pm \quad 3.3$ & $17.4 \pm$ & 3.7 & $18.0 \pm$ & 3.6 \\
\hline
\end{tabular}

All values are mean \pm standard deviation. PFA score $=$ sum of the two perceived functional ability questions. $^{*}=$ significant $(p<0.05)$ gender difference. 
Table 2. Maximal Exercise Tests Results

\begin{tabular}{|c|c|c|c|c|c|c|c|}
\hline & \multicolumn{3}{|c|}{$\begin{array}{l}\text { Male } \\
(n=38)\end{array}$} & \multicolumn{2}{|c|}{$\begin{array}{l}\text { Female } \\
(n=42)\end{array}$} & \multicolumn{2}{|c|}{$\begin{array}{l}\text { Combined } \\
(n=80)\end{array}$} \\
\hline Maximal HR & 192.6 & \pm & 6.6 & $190.8 \pm$ & 11.6 & $191.7 \pm$ & 9.6 \\
\hline Maximal HR\% & 98.2 & \pm & 3.2 & $96.1 \pm$ & 5.7 & $97.1 \pm$ & 4.8 \\
\hline Maximal RER & 1.13 & \pm & 0.03 & $1.12 \pm$ & 0.03 & $1.12 \pm$ & 0.03 \\
\hline Maximal RPE & 19.3 & \pm & 0.8 & $19.1 \pm$ & 0.8 & $19.2 \pm$ & 0.8 \\
\hline Maximal $\mathrm{VO}_{2} *$ & 52.1 & \pm & 6.5 & $43.6 \pm$ & 6.4 & $47.6 \pm$ & 7.7 \\
\hline
\end{tabular}

All values are mean \pm standard deviation. $\mathrm{VO}_{2}$ max values are in $\mathrm{mL} \cdot \mathrm{kg}^{-1} \cdot \mathrm{min}^{-1}$; Maximal HR\% is the maximal $H R$ expressed as a percent of age predicted maximal HR. $*$ significant gender differences $(p<0.05)$ 
Table 3. Step Test Results

\begin{tabular}{|c|c|c|c|c|c|c|c|}
\hline & & \multicolumn{2}{|c|}{$\begin{array}{l}\text { Male } \\
(n=38)\end{array}$} & \multicolumn{2}{|c|}{$\begin{array}{c}\text { Female } \\
(n=42)\end{array}$} & \multicolumn{2}{|c|}{$\begin{array}{c}\text { Combined } \\
(n=80)\end{array}$} \\
\hline \multirow[t]{2}{*}{ Stage 1} & $\mathrm{HR}$ & & & 136.0 & $\pm 0.0(1)$ & 136.0 & $\pm 0.0(1)$ \\
\hline & RPE & & & 7.0 & \pm 0.0 & 7.0 & \pm 0.0 \\
\hline \multirow[t]{2}{*}{ Stage 2} & $\mathrm{HR}$ & 132.3 & $\pm 17.9(3)$ & 131.0 & $\pm 15.9(10)$ & 131.3 & $\pm 15.6(13$ \\
\hline & RPE & 7.7 & \pm 1.5 & 9.4 & \pm 1.9 & 9.0 & \pm 1.9 \\
\hline \multirow[t]{2}{*}{ Stage 3} & $\mathrm{HR}$ & 126.0 & $\pm 9.7(26)$ & 130.7 & $\pm 12.8(33)$ & 128.6 & $\pm 11.7(59$ \\
\hline & RPE & 9.9 & \pm 1.9 & 10.0 & \pm 1.7 & 9.9 & \pm 1.8 \\
\hline \multirow[t]{2}{*}{ Stage 4} & $\mathrm{HR}$ & 136.5 & $\pm 12.5(36)$ & 142.2 & $\pm 12.6(36)$ & 139.4 & $\pm 12.8(72$ \\
\hline & RPE & 11.7 & \pm 2.1 & 11.8 & \pm 1.6 & 11.7 & \pm 1.8 \\
\hline \multirow[t]{2}{*}{ Stage 5} & $\mathrm{HR}$ & 149.5 & $\pm 9.5(25)$ & 149.3 & $\pm 7.4(18)$ & 149.4 & $\pm 8.6(43$ \\
\hline & RPE & 13.4 & \pm 1.9 & 13.4 & 1.9 & 13.4 & \pm 1.9 \\
\hline \multirow[t]{2}{*}{ Stage 6} & $\mathrm{HR}$ & 151.0 & $\pm 5.7(4)$ & 153.3 & $\pm 4.6(3)$ & 152.0 & $\pm 5.0(7)$ \\
\hline & RPE & 16.0 & \pm 2.1 & 13.6 & \pm 2.8 & 15.0 & \pm 2.6 \\
\hline \multicolumn{2}{|c|}{ Ending RPE } & 13.3 & $\pm 2.2(38)$ & 12.8 & $\pm 1.8(42)$ & 13.0 & $\pm 2.0(80$ \\
\hline \multicolumn{2}{|c|}{ Ending HR (bpm) } & 151.6 & $\pm 6.3(38)$ & 152.4 & $\pm 5.4(42)$ & 152.0 & $\pm 5.8(80$ \\
\hline \multicolumn{2}{|c|}{ Ending HR (\%Predicted HRmax ) } & 77.3 & $\pm 2.9(38)$ & 76.8 & $\pm 2.7(42)$ & 77.0 & $\pm 2.8(80$ \\
\hline \multicolumn{2}{|c|}{ Ending HR (\% of HRmax) } & 78.6 & $\pm 3.4(38)$ & 80.3 & $\pm 5.4(42)$ & 79.5 & $\pm 4.6(80$ \\
\hline
\end{tabular}

All values are mean \pm standard deviation $(N)$. Ending RPE and ending HR (bpm) are the RPE and HR during the final stage of the step test, respectively. Ending HR (\%Predicted HRmax) is the HR at the end of the step test expressed as a percent of age predicted maximal HR. Ending HR (\% of HRmax) is the HR at the end of the step test expressed as a percent of the actual maximal HR measured during the graded maximal exercise test. 
Table 4. Regression Model to Predict $\mathrm{VO}_{2}$ max from Step Test Data

\begin{tabular}{|c|c|c|c|c|}
\hline & & Beta & Beta Weight & $p$ value \\
\hline \multicolumn{2}{|c|}{ Intercept } & 45.938 & & $<0.0001$ \\
\hline \multicolumn{2}{|l|}{ Gender } & 9.253 & 0.606 & $<0.0001$ \\
\hline \multicolumn{2}{|l|}{$\mathrm{Kg}$} & -0.140 & -0.256 & $<0.0001$ \\
\hline \multicolumn{2}{|l|}{ PFA } & 0.670 & 0.315 & $<0.0001$ \\
\hline \multicolumn{2}{|c|}{ Final Step Rate } & 0.429 & 0.248 & 0.0004 \\
\hline \multicolumn{2}{|c|}{ 45s Recovery HR } & -0.149 & -0.238 & $<0.0001$ \\
\hline $\mathrm{R}$ & \multicolumn{2}{|l|}{0.90} & & \\
\hline SEE & \multicolumn{2}{|c|}{$3.43 \mathrm{~mL} \cdot \mathrm{kg}^{-1} \cdot \mathrm{min}^{-1}$} & & \\
\hline$R_{\text {PRESS }}$ & \multicolumn{2}{|l|}{0.88} & & \\
\hline $\mathrm{SEE}_{\text {PRESS }}$ & \multicolumn{2}{|c|}{$3.57 \mathrm{~mL} \cdot \mathrm{kg}^{-1} \cdot \mathrm{min}^{-1}$} & & \\
\hline
\end{tabular}


Figure 1. Determining the starting step height.

\begin{tabular}{|c|c|}
\hline $\begin{array}{c}\text { Participant's } \\
\text { Height }(\mathrm{cm})\end{array}$ & $\begin{array}{c}\text { Starting Step } \\
\text { Height (in) }\end{array}$ \\
\hline Under 148 & 10 \\
\hline $148-173.5$ & 12 \\
\hline $173.5-200.5$ & 14 \\
\hline Over 200.5 & 16 \\
\hline
\end{tabular}


Figure 2. Determining the starting step rate (steps/min)

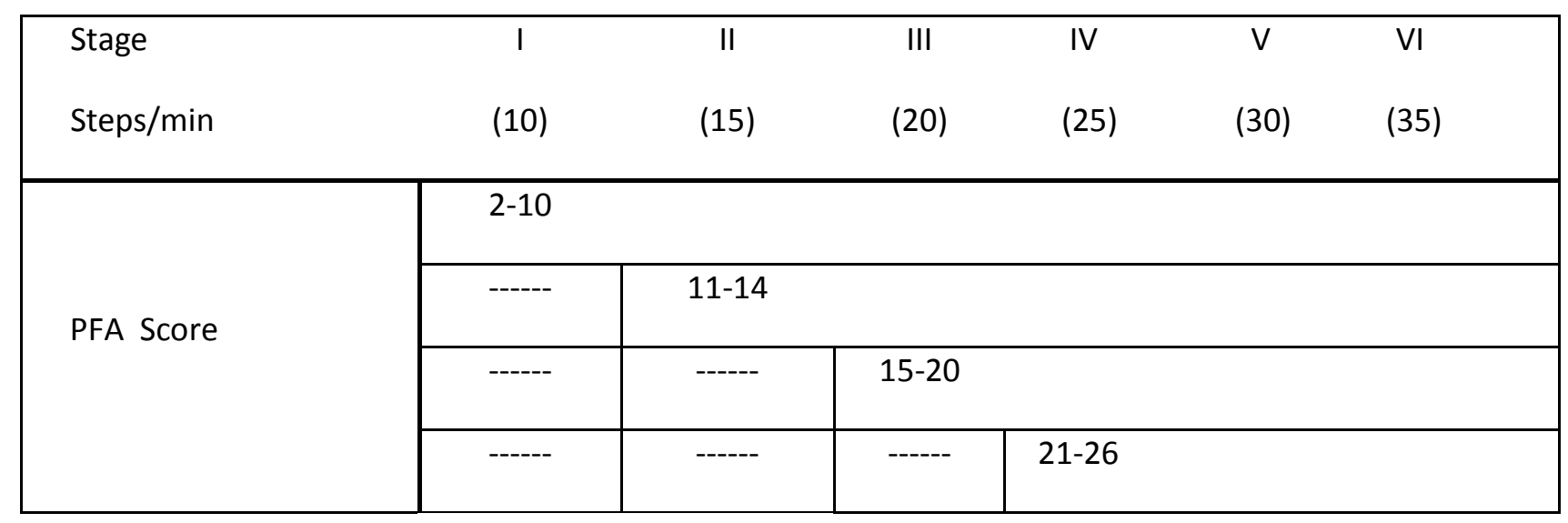


Figure 3. Scatter plot of predicted $\mathrm{VO}_{2}$ max versus measured $\mathrm{VO}_{2}$ max.

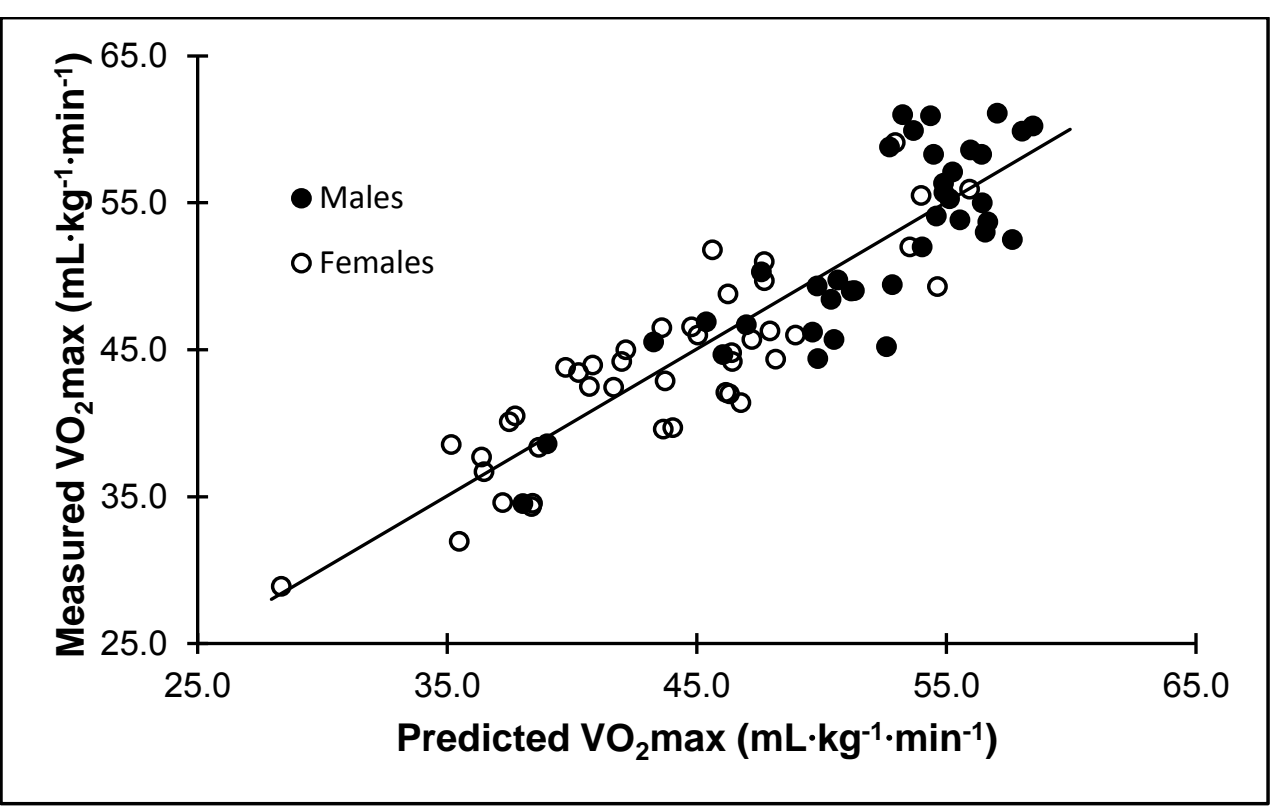




\section{References}

American College of Sports Medicine. (2010). ACSM's guidelines of exercise testing and prescription (8th ed.) Philadelphia: Lipincott Williams \& Wilkins.

Bailey, D. A., Shephard, R. J., \& Mirwald, R. L. (1976). Validation of a self administered home test of cardiorespiratory fitness. Canadian Journal of Applied Sport Sciences, 1, 67-68.

Bradshaw, D. I., George, J. D., Hyde, A, LaMonte, M. J., Vehrs, P. R., Hager, R. L., Yanowitz, F. G. (2005). An accurate $\mathrm{VO}_{2}$ max nonexercise regression model for 18-65-year-old adults. Research Quarterly for Exercise and Sport, 76(4), 426-432.

Brooks, G. A., Fahey, T. D., \& Baldwin, K. M., (2005) Exercise Physiology. Human Bioenergetics and its Applications $4^{\text {th }}$ ed. New York, NY: McGraw-Hill.

Brouha, L., Fradd, N. W., \& Savage, B. M. (1944). Studies in physical efficiency of college students. Research Quarterly, 15, 211-224.

Buckley, J. P., Sim, J., Eston, R. G., Hession, R., Fox., R. (2004). Reliability and validity of measures taken during the Chester step test to predict aerobic power and to prescribe aerobic exercise. British Journal of Sports Medicine, 38, 197-205.

Culpepper, M. I., \& Francis, K. T. (1987). An anatomical model to determine step height in step testing for estimating aerobic capacity. Journal of Theoretical Biology, 129, 1-8.

Draper, D. O., \& Jones, G. L. (1990). The 1.5 mile run revisited—an update in women's times. Journal of Physical Education, Recreation and Dance, 62(7), 78.

Francis, K., \& Brasher, J. (1992). A height-adjusted step test for predicting maximal oxygen consumption in males. Journal of Sports Medicine \& Physical Fitness, 32(3), 282-287.

Francis, K., \& Culpepper, M. (1989). Height-adjusted, rate-specific, single-stage step test for predicting maximal oxygen consumption. Southern Medical Journal, 82, 602-606. 
George, J. D., Paul, S. L., Hyde, A., Bradshaw, D. I., Vehrs, P. R., Hager, R. L., \& Yanowitz, F. D. (2009). Prediction of maximum oxygen uptake using both exercise and non-exercise data. Measurement in Physical Education and Exercise Science, 13(1), 1-12.

George, J. D., Stone, W. J., \& Burkett, L. N. (1997). Non-exercise $\mathrm{VO}_{2}$ max estimation for physically active college students. Medicine and Science in Sports and Exercise, 29(3), 415-423.

George, J. D., Vehrs, P. R., Allsen, P. A., Fellingham, G. W., \& Fisher, A. G. (1993). VO 2 max estimation from a submaximal 1-mile track jog for fit college-aged individuals. Medicine and Science in Sports and Exercise, 25, 401-406.

Heil, D.P., Freedson, P.S., Ahlquist, L.E., Price, J., and Rippe, J.M. (1995) Nonexercise regression models to estimate peak oxygen consumption. Medicine and Science in Sports and Exercise, 27, 599-606.

Jackson, A. S., Blair, S. N., Mahar, M. T., Wier, L. T., Rossand, R. M., \& Stuteville, J. E. (1990). Prediction of functional aerobic capacity without exercise testing. Medicine and Science in Sports and Exercise, 22(6), 863-870.

Keen, E. N., \& Sloan, A. W. (1958). Observations on the Harvard step test. Journal of Applied Physiology, 13(2), 241-243.

Leger, L. A., Mercier, D., Gadoury, C., \& Lambert, J. (1988) The multistage 20 metre shuttle run test for aerobic fitness. Journal of Sports Sciences, 6(2), 93-101.

McArdle, W. D., Katch, F. I., Pechard, G. S., Jacobson, L., \& Ruck, S. (1972). Reliability and interrelationships between maximal oxygen intake, physical work capacity and step test scores in college women. Medicine and Science in Sports and Exercise, 4, 182-186. 
Nielson, D.E., George, J.D., Vehrs, P.R., Hager, R.L. \& Webb, C.V. (2010). Predicting VO2max in college-aged participants using cycle ergometry and perceived functional ability. Measurement in Physical Education and Exercise Science, 14(4), 252-264.

Noble, B. J., Borg, G. A. V., \& Jacobs, I. (1983) A category-ratio perceived exertion scale: Relationship to blood and muscle lactates and heart rate. Medicine and Science in Sports and Exercise, 15(523-528).

Petrella, R. J., Koval, J. J., Cunningham, D. A., \& Paterson, D. H. (2001). A self-paced step test to predict aerobic fitness in older adults in the primary care clinic. Journal of American Geriatrics Society, 49, 632-638.

Rhyming, I. (1954). A modified Harvard step test for the evaluation of physical fitness. Arbeitsphysiologie, 15, 235-250.

Santo, A. S., \& Golding, L. A. (2003). Predicting maximal oxygen uptake from a modified 3minute step test. Research Quarterly for Exercise and Sport, 74(1), 110-115.

Siconolfi, S. F., Lasater, T. M., Snow, R. C., and Carleton, R. A. (1985). Self-reported physical activity compared with maximal oxygen uptake. American Journal of Epidemiology, 122, 101-105.

Siconolfi, S. F., Garber, C. E., Lasater, T. M., \& Carleton, R. A. (1985). A simple, valid step test for estimating maximal oxygen uptake in epidemiologic studies. American Journal of Epidemiology, 121(3), 382-390.

Sykes, K. (1998) Chester step test: Resource pack (Version 3). Cheshire, UK: Chester College of Higher Education.

Tanaka, H., Monahan, K. D., \& Seals, D. R. (2001). Age-predicted maximal heart rate revisited. Journal of the American College of Cardiology, 37(1), 153-156. 
Watkins, J. (1984). Step tests of cardiorespiratory fitness suitable for mass testing. British Journal of Sports Medicine, 18(2), 84-89.

Watkins, J., \& Ewing, B. (1984). The effects of practice and method of scoring on performance in a step test suitable for use in schools. Scottish Journal of Physical Education 12, 12 -17. 
Personalizing the Step Test

Prospectus

by

Catherine Webb

Brigham Young University

December 2009 


\section{Chapter 1}

\section{Introduction}

Cardiorespiratory fitness is described as the endurance component of physical fitness. It involves the health and function of the heart, lungs, circulatory system, and skeletal muscles. Cardiorespiratory fitness represents the body's ability to exchange oxygen (and carbon dioxide) in the lungs and the ability to deliver that oxygen to the active skeletal muscles where it is utilized in aerobic metabolism for the production of energy (Brooks, Fahey \& Baldwin 2005).

The single best measure of cardiorespiratory fitness is maximal oxygen uptake $\left(\mathrm{VO}_{2} \mathrm{max}\right)$, or the maximal amount of oxygen used during vigorous exercise (Brooks, Fahey \& Baldwin 2005). However, direct measurement of $\mathrm{VO}_{2} \max$ is time consuming, requires the use of costly equipment and trained personnel, and is thus generally reserved for laboratory settings. Due to the limitations associated with the direct measurement of $\mathrm{VO}_{2} \mathrm{max}$, a variety of submaximal and maximal exercise tests have been developed to predict cardiorespiratory fitness in the laboratory or in the field. In addition, questionnaires have been used as a tool for participants to self-report their current level of physical activity (PA-R; Jackson et al., 1990) or perceived functional ability (PFA; George et al., 2009) to exercise for one or three miles. The PA-R and PFA scores have been used to develop non-exercise predictions of cardiorespiratory fitness or provide an additional independent variable in prediction equations that estimate $\mathrm{VO}_{2} \mathrm{max}$ from data gathered during a submaximal exercise test.

The assessment of $\mathrm{VO}_{2}$ max may facilitate the development of a safe and effective exercise program. Knowledge of $\mathrm{VO}_{2}$ max can also be used to educate participants about their current fitness level compared to health, gender or age-related norms, motivate participants to set appropriate goals to maintain or increases their current levels of physical activity, and to assess 
the participant's potential risk of cardiovascular disease. Assessments of cardiorespiratory fitness can also be used to determine the effectiveness of a training program.

Suitable locations to assess cardiorespiratory fitness include community fitness centers, public schools, colleges and universities, military settings, corporate wellness centers, and in the home. Many of the field tests available to predict $\mathrm{VO}_{2}$ max using walking, jogging or running protocols are often too strenuous or are based on performance and thus may not provide accurate predictions of $\mathrm{VO}_{2}$ max. These tests require a high degree of motivation, and often have little educational value for the participant because they do not represent the intensity of exercise that would normally be undertaken. To be efficacious, the prediction of cardiorespiratory fitness should involve an exercise test that is simple to administer, timely, applicable to individuals of different fitness levels, and provide accurate and reliable estimates of $\mathrm{VO}_{2} \max$.

Step tests have long been used to estimate cardiorespiratory fitness (Brouha, Fradd, \& Savage 1944). During the step test, a participant usually steps up and down on a bench of a specific height for 3-5 minutes at a specific cadence or step rate. Heart rate during the recovery period is typically used to estimate cardiorespiratory fitness. The step test is a simple, quick, and inexpensive submaximal exercise test. However, previously developed step tests have been poor predictors of cardiorespiratory fitness, or are not well suited to the participant because the required step height or step rate are not matched to the individual's fitness level (Siconolfi et al., 1985; Brouha, Fradd \& Savage, 1944). To the best of our knowledge, a personalized step test has yet to be developed. A step test that has a personalized bench height and step rate may increase the validity of the test in estimating $\mathrm{VO}_{2} \max$. Information such as PFA could be used to determine an appropriate initial step height and step rate based on perceived physical fitness levels. Heart rate data from the personalized step test could be used along with other variables 
such as step height, step rate, body mass index (BMI), PFA, PA-R, and gender to develop a regression equation which would more accurately predict $\mathrm{VO}_{2} \max$.

\section{Statement of Purpose}

The purpose of this study is to develop a personalized step test and a regression equation that can be used with step test data to accurately predict $\mathrm{VO}_{2}$ max in males and females 20 to 29 years of age. A personalized step test may be used in a variety of settings to quickly, yet accurately estimate cardiorespiratory fitness.

\section{Hypotheses}

Research hypothesis: The predicted $\mathrm{VO}_{2}$ max from the multiple regression equation using various predictive variables such as: age, gender, BMI, PFA, PA-R and personalized step test heart rates will have a significant positive correlation with directly measured $\mathrm{VO}_{2}$ max.

Null hypothesis: There is no significant positive correlation between the directly measured $\mathrm{VO}_{2}$ max and predicted $\mathrm{VO}_{2}$ max from a multiple regression equation using age, gender, BMI, PFA, PA-R and personalized step test heart rates.

\section{Definition of Terms}

$\mathrm{VO}_{2} \mathrm{max}$ - The highest level of oxygen that is consumed during strenuous aerobic exercise, measured during a maximal graded exercise test on the treadmill.

Step height - The height of the step test bench.

Step rate - The number of complete step-ups and step-downs taken during one minute. One complete step sequence includes step-up, step-up, step-down, step-down.

\section{Assumptions}

1. The age-predicted heart rate max is an accurate indicator of maximal heart rate. 
2. The participants reach a maximal level of performance during the graded exercise test (GXT).

\section{Delimitations}

The results are only applicable to the study population: healthy males and females between the ages of 18 and 29, the majority of which are Caucasian.

\section{Limitations}

According to the pre-exercise screening questionnaire, individuals with health concerns where the safety of a maximal treadmill exercise test is questioned will not be participants. 


\section{Chapter 2}

\section{Review of Literature}

Based on scientific research, the American College of Sports Medicine (ACSM), American Heart Association (AHA), and the Department of Health and Human Services (DHHS) have published physical activity guidelines for Americans (Pate, et al., 1995; Haskell, et al., 2007; Centers for Disease Control and Prevention, 2009; U.S. Department of Health and Human Services, 2009a). The intent of the national physical activity guidelines is to increase the overall physical activity levels of the population, thereby reducing the risk of diseases related to physical inactivity and obesity. Increasing the physical activity levels of Americans has become a public health initiative because the majority of adults in the United States are "effectively sedentary" and every year in the United States alone, as many as 250,000 people die due to lack of regular physical activity (Myers, 2003).

Physical activity improves health by reducing body mass, reducing blood pressure, improving cholesterol levels and increasing cardiorespiratory fitness (Myers, 2003). Cardiorespiratory fitness is defined as the amount of oxygen the body can utilize during strenuous aerobic exercise (Brooks, Fahey \& Baldwin 2005). Improving cardiorespiratory fitness decreases the risks for cardiovascular disease, coronary heart disease, stroke, obesity, and type 2 diabetes.

Prior to starting an exercise program, an assessment of one's cardiorespiratory fitness can be useful when designing a safe and effective exercise program. Information about one's cardiorespiratory fitness is also educational, motivational and useful for determining a baseline to which future measurements can be compared. The most accurate measure of cardiorespiratory 
fitness is the maximum amount of oxygen a person is able to consume during strenuous exercise, also known as maximum oxygen uptake, or $\mathrm{VO}_{2} \max$ (Santo \& Golding, 2003).

\section{Measuring $\mathrm{VO}_{2} \max$}

The measurement of $\mathrm{VO}_{2} \mathrm{max}$ is typically reserved for a laboratory setting since it requires the use of expensive equipment and trained personnel. $\mathrm{VO}_{2} \mathrm{max}$ is measured during a maximal graded exercise test (GXT) which is not well tolerated by the unfit, overweight or elderly due to the demanding, maximal effort required by the participant (Santo \& Golding, 2003). The need for costly instrumentation and trained personnel make maximal GXTs impractical for large groups or those in remote areas. As a result, many exercise tests have been developed to predict $\mathrm{VO}_{2}$ max from less demanding, submaximal exercise tests that can be performed in the field (Rhyming, 1954; Francis \& Brasher, 1992; Francis \& Culpepper, 1989). There are a wide variety of submaximal tests including a 1-mile jog (George, Vehrs, Allsen, Fellingham \& Fisher 1993) a 1.5-mile run (Draper \& Jones 1990; Jackson et al., 1981), a 12minute run-walk test (Cooper et al., 1975), a 3-mile run (Jackson et al., 1990), a walk-jog-run test (George et al., 2009) a 20 m shuttle run (Leger, Mercier, Gadoury, \& Lambert, 1988) and step tests (Kasch, 1961; Siconolfi, Garber, Lasater \& Carleton, 1985). The majority of the tests can be performed individually or in large groups. Most importantly, the submaximal tests typically do not require strenuous levels of exertion, and therefore are generally safer for the participant.

\section{Predicting $\mathrm{VO}_{2}$ max with $P A-R$ and $P F A$}

$\mathrm{VO}_{2}$ max can also be predicted using multiple regression equations that use non-exercise data. Assessing physical activity levels through self-reported data may be more time and cost efficient than exercise testing (Bowles, Fitzgerald, Morrow, Jackson \& Blair, 2004). Information 
from self-reported data may be historical (within the previous year) or hypothetical, and is often used for epidemiological research (Bowles et al., 2004). Multiple studies suggest that selfreported exercise data may be useful in generating a multiple regression equation to predict $\mathrm{VO}_{2} \max$ (Bowles et al., 2004; Jackson et al., 1990). Non-exercise models include variables such as age, BMI, gender, self reported physical activity levels, and PFA (George et al., 2009; George, Stone, \& Burkett, 1997). The responses of 12,225 individuals to a survey were used to develop a regression equation using self-reported levels of walking, jogging and running, age, and frequency of sweating to predict total treadmill time (time on treadmill until voluntary exhaustion) with a multiple correlation coefficient of 0.65 (Kohl, Blair, Paffenbarger, Marcera \& Kronenfeld, 1988). In 1990, Jackson et al. reported that a prediction model consisting of age, gender, BMI, percent body fat, and self-reported physical activity to predict $\mathrm{VO}_{2}$ peak $(R=.81)$ performed better than the well established Astrand submaximal tests. Jackson et al. (1990) used the PA-R for the self-reported physical activity component, which asks the participant to rate their activity level in the last six months (Ross \& Jackson, 1990). In 1997 George, et al. increased the predictive accuracy $(R=.86)$ by including in the regression equation the variable of the participant's PFA to walk, jog or run 1-and 3-mile distances (see Appendix A). They also modified the PA-R to better accommodate high fit individuals by increasing the scale to 10 (see Appendix B) (George et al., 1997). In 2009 George et al., generated a new regression equation using the PFA and other non-exercise data (age, gender, BMI, and PA-R) as predictor variables along with exercise data (heart rate and work rate) from a submaximal treadmill test. The accuracy of the prediction equation that included both exercise and non-exercise variables exceeded $(R=.94)$ that of previous prediction equations using either exercise or non-exercise data. 
To date, the PA-R and PFA predictor variables have not been employed in a $\mathrm{VO}_{2}$ max regression model with exercise data using a step test. In addition, as indicators of current levels of physical activity and cardiorespiratory fitness, the PA-R and PFA variables have not been used to determine the starting intensity of the step test. Step Test

The step test has emerged as a popular and common predictor of $\mathrm{VO}_{2}$ max $($ Keen $\&$ Sloan, 1958). The step test is easy to administer with minimal equipment and requires moderate levels of intensity and little time commitment of the participant (Francis \& Culpepper, 1989). Due to its popularity, several versions of step tests exist. Generally, participants step up and down in a four-step sequence on a bench or stairs 20-50 cm high, keeping time with a predetermined beat on a metronome. The step provides a physical challenge which raises the particpants' heart rate to a submaximal level. The duration of the test is usually 3-5 minutes, after which recovery heart rate is recorded. Room temperature has been found to affect the results of the step test, as well as bench height, stepping rate, leg length, and body composition (Chen et al., 2006; Francis \& Culpepper, 1989; Keen \& Sloan, 1958). The regression equations for predicting $\mathrm{VO}_{2} \max$ from the step test have included predictor variables such as recovery heart rate, exercise heart rate, test duration, age, gender, and body weight (McArdle, Katch, Pechard, Jacobson \& Ruck, 1972; Shephard, 1980; Siconolfi et al., 1985). Many of the earlier step tests did not predict $\mathrm{VO}_{2} \mathrm{max}$, but simply estimated fitness levels by assigning the participant into a fitness category. Step tests can be divided into two groups, the single-stage and the multi-stage step tests. 


\section{Single-Stage Step Test}

Although the single-stage step test is less personalized, it is relatively short in duration (usually 3-5 minutes) and often less complex than the multi-stage step tests. The single stagestep test is ideal for use in large groups or simultaneous testing of individuals. The first step test was developed at Harvard by Brouha et al. (1944). The Harvard Step Test required participants to step up 30 times/min on a $50.8 \mathrm{~cm}$ bench (the same as many bleachers) for five minutes or until they stopped from exhaustion. The sum of three post-exercise pulse counts was used to estimate $\mathrm{VO}_{2}$ max. Although this step test was valid for young athletic males, it is too strenuous for the untrained and aged (Watkins, 1984). In addition, the test is not suitable for those with a short stature due to the high bench height which often results in localized leg fatigue and early termination of the test. For those who are short in stature, the step-test appeared to be a measure of muscle endurance rather than cardiovascular fitness (Watkins, 1984; Datta, Chatterjee \& Roy, 1974).

Following the development of the Harvard Step Test, other less intense step tests were created. Rhyming (1954) used a bench height of $33 \mathrm{~cm}$ for females and $40 \mathrm{~cm}$ for males and a stepping rate of $22.5 \mathrm{step}-\mathrm{ups} / \mathrm{min}$. Kasch (1961) validated a step test using a $30.5 \mathrm{~cm}$ bench and 24 step-ups/min performed for three minutes. The recovery heart rate that was counted for one minute following the test was used to determine the participant's cardiorespiratory fitness category. The test was adopted by the Young Men's Christian Association (YMCA) in 1970 and became known as the YMCA 3- minute Step Test (Santo \& Golding, 2003). Its short duration and simple protocol are ideal for large group testing and it places participants into a fitness category. However this test does not provide an estimate of $\mathrm{VO}_{2} \mathrm{max}$ (Kasch, 1961). 
In 1972, McArdle developed what became known as the Queens College Step Test, with

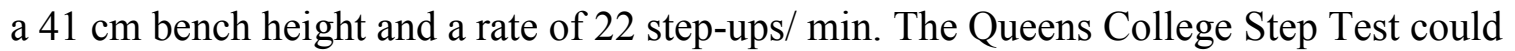
reasonably be used in the general population because of the lower bench height and slower step rate. The Queens College Step Test does have an accompanying regression model and could predict $\mathrm{VO}_{2}$ max with a correlation coefficient of 0.75 (McArdle et al., 1972).

\section{Multistage Step Test}

Multistage step tests allow for a rest period between stages in which to record heart rate and determine if and how the individual should proceed. The advantage of this is a more personalized test where the individual progresses through subsequent stages based on his or her heart rate response to the previous stage. Compared to single-stage step tests, multistage step tests often have greater validity because participants reach a predetermined percentage of their age-predicted maximal heart rate. A disadvantage of multistage step tests is the longer duration of the test, especially for fit individuals. Because of the differences in the length of the test between individuals and the progression of the test based on heart rate, multistage step tests are generally not conducive to a group setting.

One of the first multistage step tests was the Canadian Home Fitness Test (Bailey, Shephard \& Mirwald, 1976), designed to use the typical Canadian stair step of an 8 inch height. Participants used a six-beat rhythm to step up and down two stairs for up to three stages of three minutes each. The stepping rate during each stage was determined by age, and the pulse count taken 5-15 seconds into recovery determined if the subject would proceed to the next stage. Although the results of the test were not used to predict $\mathrm{VO}_{2} \mathrm{max}$, an advantage of the Canadian Home Fitness Test was the ease of categorizing one's fitness level. The fitness category of the participant was categorized based on the last stage completed. If the participant completed only 
the first stage, fitness level was categorized as very poor. If the participant completed the second or third stage, the fitness level was categorized as poor or good, respectively (Bailey et al., 1976; Shephard, 1980). Although the Canadian Home Fitness Test is convenient for home use or to screen fitness level, the lack of an actual predicted $\mathrm{VO}_{2}$ max value makes it less applicable for those who are interested in determining their $\mathrm{VO}_{2}$ max or monitoring improvement in cardiorespiratory fitness as a result of a physical activity or exercise program.

The Siconolfi Step Test (1985) was successful in estimating $\mathrm{VO}_{2}$ max using a three-stage protocol. The test uses a $25.4 \mathrm{~cm}$ bench height and step rates of 17, 26 and 34 step-ups/min for stages one, two and three, respectively. The exercise heart rate was measured three times during the last minute of each stage. If the average of the three heart rates did not reach 65 percent of age-predicted maximal heart rate at the end of the stage, the participant would proceed to the next stage following a one-minute rest. The correlation coefficients between estimated and measured $\mathrm{VO}_{2}$ max range from 0.89 to 0.98 depending on the age group (Siconolfi et al., 1985).

One recent multistage step test for the elderly was effective in predicting $\mathrm{VO}_{2}$ max after letting participants select their own stepping pace for slow, normal and fast stages (Petrella, Koval, Cunningham \& Paterson, 2001). The time to complete 20 step-ups was recorded for each stage, as well as an immediate post-exercise heart rate. The advantages to this method are the decrease in unnecessary testing time and an increase in safety for the elderly or unfit.

The Chester Step Test contains five stages of two minutes each, and the participants are assigned a step height based on fitness level, age and height. Every two minutes the pace is increased until the participant reaches $80 \%$ of his/her age predicted maximal heart-rate (Buckley, Sim, Eston, Hession \& Fox 2004). The step test is unique due to the personalization in step height, yet it often takes 8-10 minutes to complete, and the first stages become irrelevant when 
graphing the heart-rates. In addition, the older equation used to predict maximal heart-rate of 220 minus age and the $\mathrm{VO}_{2}$ max estimation of each stage were potential sources of error (Buckley et al., 2004).

Recovery Heart Rate as an Indicator of Cardiorespiratory Fitness

During light- to moderate-intensity aerobic exercise, there is a linear relationship between heart-rate response and oxygen consumption (Watkins, 1984). The heart-rate response during the recovery period immediately following exercise can be used as an indicator of an individual's cardiorespiratory fitness level. Generally, the faster one's heart rate returns to a resting heart-rate following exercise, the higher the fitness level. Recovery heart-rate is often used instead of exercise heart-rate to predict $\mathrm{VO}_{2}$ max because it is easier to obtain in a field setting or in large groups, but its effectiveness in predicting $\mathrm{VO}_{2}$ max depends on the time it is recorded following exercise (Watkins, 1984). As indicated above, the Harvard step test uses the sum of three pulse counts from 1-1:30, 2-2:30 and 3-3:30 minutes into recovery to estimate cardiorespiratory fitness (Brouha, 1944). The Kasch step test uses heart-rate for 5-65 seconds into recovery to predict $\mathrm{VO}_{2} \max$ ( Kasch, 1961). In 1972, McArdle et al. determined that recovery heart-rate recorded from 5 to 20 seconds into recovery had the highest validity coefficient $(R=-.76)$ when using the Queens College Step Test. Heart-rates recorded 60-75 seconds into recovery were so highly correlated with earlier heart-rate recordings $(R=.94)$, that they were deemed unnecessary (McArdle et al., 1972).

Watkins and Ewing (1984) tested the reliability of the step test using six different test scores from three heart-rate recordings. The time for 30 heart beats was recorded, starting at 5, 60 and 120 seconds into recovery (S1, S2, S3). The three other scores (S4, S5, S6) were the sum of one and two, two and three, and the sum of all three, respectively. The highest correlation 
coefficient was from S1 $(R=0.94)$, while the correlation coefficients from S2, S3, S4, S5 and S6 $(R=0.74,0.55,0.87,0.67$ and 0.79 , respectively) were much lower. In line with McArdle et al. (1972), Watkins and Ewing (1984) found the heart rate from the first 20 seconds of recovery to be the most effective in predicting $\mathrm{VO}_{2} \max$.

Step tests have also been successful in estimating $\mathrm{VO}_{2} \max$ when using the exercise heart rate (Rhyming, 1954; Siconolfi et al., 1985). Benefits to using the exercise heart-rate are a reduction in test taking time and an improved estimation of the exercise workload. Rhyming (1954) determined the pulse count from the radial artery every 15 seconds of a five-minute step test, and found that in most cases the pulse rate maintained a steady value after two minutes. If no steady value could be obtained, the highest heart-rate value was recorded. Recovery heart rate was also recorded 1:00 to 1:30 into recovery. The high correlation $(R=0.96 \pm 0.01)$ between exercise pulse rate and recovery heart rate values for the same participant indicates that exercise heart rate can be used in place of recovery heart rate and vice versa. However, when comparing the exercise pulse rate with the 1:00- 1:30 minute recovery heart rate in 61 different participants, the correlation was much lower $(R=0.77 \pm 0.04)$ (Rhyming, 1954). Rhyming notes that if the workload is light, the recovery heart rate will quickly return to resting values and the 1:00-1:30 minute recovery heart rate may not be an effective indicator of the workload (Rhyming, 1954).

Siconolfi et al (1985) recorded the heart rate at 2:30, 2:45, and 3:00 (also the end of that stage). If the average of the three heart rates was not greater than or equal to 65 percent of the age-predicted maximum, then the participant moved on to the next stage, where heart rate was recorded as in the first stage. By measuring the exercise heart rate and adjusting accordingly, this step test ensured that the submaximal workload was eliciting an exercise response. The inexpensive equipment used and the low 65 percent threshold make this step test appealing for 
use with the general population at home or in the lab. The regression equation from the step test predicted $\mathrm{VO}_{2}$ max with high correlation coefficients ranging from 0.89 to 0.98 for the age groups (SEE ranged from 0.14 to $0.21 \mathrm{~L} / \mathrm{min}$ ) (Siconolfi et al., 1985).

Leg Length and the Step Test

Some research suggests that a participant's leg length is a factor in predicting $\mathrm{VO}_{2}$ max from the step test because step height influences biomechanical efficiency and heart rate (Ashley, Smith \& Reneau, 1997; Francis \& Culpepper, 1989; Shahnawaz, 1978;). Keen and Sloan (1958) reported that the high bench height of the Harvard Step Test may impose a disadvantage for those individuals with shorter leg lengths. Shahnawaz (1978) measured $\mathrm{O}_{2}$ consumption on ten subjects while they preformed the step test on several different bench heights. The lowest $\mathrm{O}_{2}$ consumption was found when the bench height was 50 percent of the leg length, and a significant relationship between leg length and $\mathrm{O}_{2}$ consumption during the step test existed. However the results may be limited due to the small number of participants. Culpepper \& Francis (1987) developed an equation to determine bench height based on hip angle, in which the bench height resulting in the hip angle of 73.3 degrees was found to give the best correlations of recovery heart rate to $\mathrm{VO}_{2} \max$. In 1989 they tested the use of this equation on women, and in 1992 on men, performing three different step tests at stepping rates of 22, 26 and $30 \mathrm{step}$-ups $/ \mathrm{min}$. The correlation coefficients between recovery heart rate taken 5-20 seconds post-exercise and $\mathrm{VO}_{2}$ max from both studies were very similar $(R=.72, .81, .72$ for rates of 22,26 and 30 stepups/min) and were comparable to those from step tests using a standardized bench height for men and women (Francis \& Brasher, 1992; Francis \& Culpepper, 1989; McArdle et al., 1972). The results of these studies may be also limited by the lack of comparison with an unmodified step test of the same type. A recent study modified the Queens College Step Test by setting the 
bench height so the knee angle was 90 degrees (Ashley et al., 1997). Compared with the normal Queens College Step Test, it concluded that leg length is only a significant factor when the bench is $41 \mathrm{~cm}$ (16 inches) high or higher, and step tests using a step height based on leg length do not more accurately predict $\mathrm{VO}_{2} \max$ than those using a standardized bench height (Ashley et al., 1997; Watkins 1984). Many step test benches are below $41 \mathrm{~cm}$, and therefore should not have inaccurate results from different leg lengths.

\section{Body Composition and the Step Test}

A recent study using a modified YMCA Step Test reported that body composition may affect recovery heart rate obtained following submaximal exercise testing, and therefore limit the prediction of $\mathrm{VO}_{2} \max$ (Santo \& Golding, 2003). A participant with excess weight would have to work harder to perform the same submaximal workload, therefore, would have a higher exercising heart rate, a higher recovery heart rate, would be assigned to a lower fitness category and would, in theory, have a lower predicted $\mathrm{VO}_{2} \mathrm{max}$. Keen and Sloan (1958), however, did not find any correlation between body weight and the fitness rating when studying 75 healthy young men 17 to 27 years of age performing the Harvard Step Test. It seems that body weight is not a limitation of the step test when using healthy and college-aged participants.

\section{Personalizing the Step Test}

It would be beneficial to develop a personalized, multistage step test that is easy to perform in a short amount of time. The step test can be personalized based on cardiorespiratory fitness level by using the PFA questionnaire (see Appendix A) to determine the starting step height and step rate of the step test. This would also eliminate unnecessary stages for the more fit participants. Personalization could further be accomplished by adjusting the stepping rate according to the heart-rate response and RPE during each stage of the step test. This would allow 
adjustments in workload based on the participant's fitness level and responses to exercise. Although this type of protocol has not been tested to date, pilot studies deem it successful. It is hypothesized that a submaximal step test using this protocol would be able to estimate $\mathrm{VO}_{2} \max$ with increased accuracy within a reasonable testing time. A personalized step test will be more suitable for the general population than previously developed step tests. To date no step test has used PFA or PA-R as a predictor variable in the regression model to predict $\mathrm{VO}_{2}$ max nor has a step test involved PFA in determining the starting step height and/or stepping rate based on cardiorespiratory fitness level. 


\section{Chapter 3}

Methods

\section{Participants}

The participants in this study will include 50 males and 50 females, 18-29 years of age. Exclusion criteria for the participants will be known conditions that increase the risk of cardiovascular, pulmonary, or metabolic events during exercise testing. A pre-participation questionnaire (see Appendix D) will be completed by each participant to screen for exclusion criteria and exclude those participants who may be at risk during a maximal exercise test. (ACSM, 2010).

Before collection of data, approval for use of human subjects will be obtained from the Institutional Review Board (IRB) at Brigham Young University (BYU). Participants will be recruited through fliers and announcements in BYU classes. All participants will provide written informed consent prior to participation in the study. All participants who complete the study will receive $\$ 5$ cash.

\section{Procedures}

Each participant will perform a submaximal step test and a maximal GXT on the treadmill. Data collected during the step test and the recovery period following the step test will be used as independent variables to predict $\mathrm{VO}_{2}$ max measured during the GXT.

Pre-exercise testing procedures. Participants will be instructed to (a) wear comfortable clothes and shoes appropriate for running, (b) drink plenty of fluids over the 24-hour period prior to exercise testing to ensure normal hydration, (c) refrain from eating food other than water, and from using tobacco, alcohol, and caffeine for two to three hours prior to exercise testing, and (d) avoid exercise or strenuous physical activity the day of the testing. Participants will be approved 
for the exercise test after positively answering all questions from the Physical Activity Readiness Questionnaire (PAR-Q).

Participants will report to the Exercise Physiology Lab (121 Richards Building) in the Human Performance Research Center at BYU to perform the submaximal step test and the maximal GXT on the treadmill. Upon arrival, participants will provide written informed consent and complete two questionnaires that consist of a total of three questions that self-report their perceived functional ability (PFA; Appendix A) and physical activity rating (PA-R; Appendix B) (George et al., 2009; George, et al., 1997; Jackson et al., 1990).

Each participant's weight $(\mathrm{kg})$ and height $(\mathrm{cm})$ will be measured and recorded to the nearest one-tenth of a kilogram, and to the nearest one-half centimeter, respectively, using a digital weight scale (Ohaus Model CD-33, Ohaus Corporation, Pine Brook NJ, USA) and a calibrated wall scale.

Exercise testing. During the submaximal step test and the maximal GXT, heart rate will be monitored using a radiotelemetry heart-rate monitor (Polar Electro OY, Hong Kong) and rating of perceived exertion (RPE) will be monitored after each test using the Borg 15-point scale (Noble, Borg, \& Jacobs, 1983; Appendix D).

Each participant will complete a submaximal step test once. Twenty participants will repeat the submaximal step test on a second day to determine the reliability of the step test to predict $\mathrm{VO}_{2} \max$. The step test will be performed on The Step (The Step, Inc., Marietta, GA) with three, four or five sets of risers to set the step height at either 10, 12 or 14 inches, respectively. In order to develop a more accurate and valid step test, the equation by Francis and Culpepper (0.19 *participant height in $\mathrm{cm}$ ) (1989) which allows the participant to have the ideal hip angle of 73.3 is used to determine the step test step height (Table 1). In addition, to personalize the step test 
based on perceived physical fitness levels, the height of the step determined by Francis and Culpepper's equation (1989) is adjusted if the participant has a PFA score under 14, or over 20

(Figure 1). The PFA score is the sum of the responses to both PFA questions. This study uses the Chester Step Test's five, two-minute stage protocol (Buckley et al., 2004). However, in order to personalize the test, and reduce unnecessary stepping, the participant will begin the step test at either stage I, II, or III, with stepping rates of 15, 20 and 25 steps/min, respectively (Figure 2). The PFA score will determine at which stage the participant begins the test. Participants with a PFA score of 2-13, 14-20, or 21-26 begin the step test at stages I, II, or III, respectively.

The participant's resting heart-rate (after sitting quietly for 5 minutes) will be recorded prior to beginning exercise testing. The participant will familiarize themselves with the four-step sequence of up/up, down/down at the predetermined step height and stepping rate before the test. Participants will be instructed on the proper form during stepping (i.e. to keep their knees and back straight at the top) and proper form will be monitored during the test. A metronome will be used to help them keep the cadence during the familiarization and the exercise test. Following the familiarization, the exercise test will begin at the predetermined step height (Figure 1) and stepping rate (Figure 2). Each participant's 75\% of age-predicted maximal heart-rate (MHR) will be calculated using the formula, (207- (0.7*age)) (Tanaka, Monahan \& Seals, 2001). After two minutes all participants will increase the stepping rate by five steps/min and continue in like manner every two minutes until the heart-rate is equal to or greater than $75 \%$ age-predicted MHR and/or RPE is 14 (Buckley et al., 2004). Heart-rate and RPE will be recorded near the end of each two minute stage. When the heart-rate is equal to or exceeds $75 \%$ of age-predicted MHR and/or RPE is 14, the participant will finish the current stage and the test will be completed. The participants will complete two or more stages of the step test (if they are unable to complete at 
least two stages, they must restart the test at a lower height after heart-rate has returned to near resting levels). Immediately following the completion of the step-test, participants will assume a seated position and a heart-rate will be recorded every 15 seconds post-exercise for one minute. The heart-rate and RPE from the last step test stage completed, post-exercise heart-rates, step height, and final stepping rate will be used as independent variables in the statistical analysis to predict $\mathrm{VO}_{2} \max$.

After the submaximal step test, the participants will have an active and resting recovery for approximately 10 minutes until their heart-rate returns to near resting values before completing the maximal GXT. The GXT will be performed on a motor-driven treadmill (Model TMX425C, Full Vision, Inc., Newton, KS). Metabolic and ventilatory responses to exercise will be measured using a Truemax 2400 metabolic cart (Consentious Technologies, Sandy, UT). Prior to each maximal GXT, the flow meter will be calibrated at five different flow rates using a 3-L syringe and the oxygen and carbon dioxide analyzers will be calibrated using room air and a medical grade calibration gas of known concentrations. The metabolic cart will be programmed to display and print metabolic and ventilatory data every 15 seconds. Participants will be fitted with a mouthpiece, one-way breathing valve, and a nose clip to aid in the measurement of expired air and gases.

Each participant will complete a maximal GXT similar to a protocol previously described ( George et al., 2009). During the first minute on the treadmill, participants will be instructed to walk at a comfortable pace. From minute one to minute two, participants will be instructed to choose a treadmill jogging speed that they could comfortably maintain for 25-30 minutes. This treadmill speed will remain constant during the remaining stages of the exercise test. The treadmill grade will be increased starting after the third minute, $1.5 \%$ every minute until the 
subject voluntarily terminates the test due to fatigue, despite verbal encouragement. After terminating the test, participants will have a walking cool down session at a self-selected speed at level grade for any desired amount of time.

The participant's effort will be considered maximal if physical signs representative of exhaustion are obvious and at least two of the following four criteria are met: (a) maximal respiratory exchange ratio (RER) greater than or equal to 1.10 , (b) a maximal heart-rate that is no less than 15 beats below age-predicted maximal HR, (c) a RPE from the participant of 19 or 20 on Borg's RPE scale, and (d) a leveling off of $\mathrm{VO}_{2}$. Maximal heart-rate will be defined as the highest single heart-rate value during the GXT and maximal $\mathrm{VO}_{2}$ will be defined as the highest 30-s average $\mathrm{VO}_{2}$ value during the final minutes of the exercise test.

\section{Statistics}

Statistical software (SAS) will be used for all data analyses. The measured $\mathrm{VO}_{2}$ max will be entered as the dependent variable with age, gender, step height, end stepping rate, step-test ending heart-rate, step-test recovery heart-rate, PFA score, PA-R score, and any reasonable twoway interactions considered independent variables in a regression analysis to predict $\mathrm{VO}_{2} \mathrm{max}$. An alpha level of $p<0.05$ will be maintained in all analyses. 


\section{Figure 1:}

\section{Step Height}

Participant's

Height (cm)

\begin{tabular}{|l|l|}
\hline Under $148 \mathrm{~cm}$ & $10 \mathrm{in}$ \\
\hline $148-173.5 \mathrm{~cm}$ & $12 \mathrm{in}$ \\
\hline Over $173.5 \mathrm{~cm}$ & $14 \mathrm{in}$ \\
\hline
\end{tabular}

Figure 2:

\section{Starting Stepping Rate}

\begin{tabular}{|c|cccccc|}
\hline $\begin{array}{c}\text { Stage \& } \\
\text { Steps/min }\end{array}$ & I & II & III & IV & V & VI \\
\hline \multirow{3}{*}{ PFA Score } & $(15)$ & $(20)$ & $(25)$ & $(30)$ & $(35)$ & $(40)$ \\
\cline { 2 - 6 } & $2-13$ & $14-20$ & \\
\cline { 2 - 6 } & ------ & ------ & $21-26$ \\
\hline
\end{tabular}

Figure 3:

Estimated $\mathrm{VO}_{2}(\mathrm{ml} / \mathrm{kg} / \mathrm{min})$

\begin{tabular}{|c|c|c|c|c|c|c|}
\hline $\begin{array}{c}\text { Stage \& } \\
\text { Steps/min }\end{array}$ & $\begin{array}{c}\text { I } \\
(15)\end{array}$ & $\begin{array}{c}\text { II } \\
(20)\end{array}$ & $\begin{array}{c}\text { III } \\
(25)\end{array}$ & $\begin{array}{l}\text { IV } \\
(30)\end{array}$ & $\begin{array}{c}\mathrm{V} \\
(35)\end{array}$ & $\begin{array}{l}\text { VI } \\
(40)\end{array}$ \\
\hline 10 in step & 15.6 & 19.7 & 23.7 & 27.7 & 31.8 & 37 \\
\hline 12 in step & 17.4 & 22.1 & 26.7 & 31.4 & 36.0 & 41 \\
\hline 14 in step & ------ & 24.5 & 29.8 & 35.0 & 40.3 & 45 \\
\hline
\end{tabular}




\section{References}

American College of Sports Medicine. (2010). ACSM's guidelines of exercise testing and prescription (8th ed.) Philadelphia: Lipincott Williams \& Wilkins.

Ashley, C. D., Smith, J. F., \& Reneau, P. D. (1997). A modified step test based on a function of subjects' stature. Perceptual and Motor Skills, 85(3), 987-993.

Bailey, D. A., Shephard, R. J., \& Mirwald, R. L. (1976). Validation of a self administered home test of cardiorespiratory fitness. Canadian Journal of Applied Sport Sciences, 1, 67-68.

Bowles, H. R., Fitzgerald, S. J., Morrow, J. R., Jackson, A. W., \& Blair, S. N. (2004). Construct validity of self-reported historical physical activity. American Journal of Epidemiology, $160(3), 279-286$.

Brooks, G. A., Fahey, T. D., \& Baldwin, K. M., (2005) Exercise Physiology. Human Bioenergetics and its Applications $4^{\text {th }}$ ed. New York, NY: McGraw-Hill.

Brouha, L., Fradd, N. W., \& Savage, B. M. (1944). Studies in physical efficiency of college students. Research Quarterly, 15, 211-224.

Buckley, J. P., Sim, J., Eston, R. G., Hession, R., Fox., R. (2004). Reliability and validity of measures taken during the Chester step test to predict aerobis power and to prescribe aerobic exercise. British Journal of Sports Medicine, 38, 197-205.

Centers for Disease Control and Prevention. (2009). How much physical activity do adults need? Retrieved from http://www.cdc.gov/physicalactivity/everyone/guidelines/adults.html

Chen, S-M., Wang, J-S., Lee, W-C., Hou, C-W., Chen, C-Y., Laio, Y-H., Lin C-H., \& Kuo, C-H. (2006). Validity of the 3 minute step test in moderate altitude: Environmental temperature as a confounder. Applied Physiology of Nutrition and Metabolism, 31, 726730. 
Cooper, K. H., Purdy, J. G., Friedman, A., Bohannon, R. L., Harris, R. A., \& Arends, J. A. (1975). An aerobics conditioning program for the Fort Worth, Texas School District. Research Quarterly, 46(3), 345-350.

Culpepper, M. I., \& Francis, K. T. (1987). An anatomical model to determine step height in step testing for estimating aerobic capacity. Journal of Theoretical Biology, 129, 1-8.

Datta, S. R., Chatterjee, B. B., \& Roy, B. N. (1974). An improved simple exercise test for evaluation of physical fitness. Ergonomics, 17, 105-112.

Draper, D. O., \& Jones, G. L. (1990). The 1.5 mile run revisited—an update in women's times. Journal of Physical Education, Recreation and Dance, 62(7), 78.

Francis, K., \& Brasher, J. (1992). A height-adjusted step test for predicting maximal oxygen consumption in males. Journal of Sports Medicine \& Physical Fitness, 32(3), 282-287.

Francis, K., \& Culpepper, M. (1989). Height-adjusted, rate-specific, single-stage step test for predicting maximal oxygen consumption. Southern Medical Journal, 82, 602-606.

George, J. D., Paul, S. L., Hyde, A., Bradshaw, D. I., Vehrs, P. R., Hager, R. L., \& Yanowitz, F. D. (2009). Prediction of maximum oxygen uptake using both exercise and non-exercise data. Measurement in Physical Education and Exercise Science, 13(1), 1-12.

George, J. D., Stone, W. J., \& Burkett, L. N. (1997). Non-exercise $\mathrm{VO}_{2}$ max estimation for physically active college students. Medicine and Science in Sports and Exercise, 29(3), 415-423.

George, J. D., Vehrs, P. R., Allsen, P. A., Fellingham, G. W., \& Fisher, A. G. (1993). VO max $^{2}$ estimation from a submaximal 1-mile track jog for fit college-aged individuals. Medicine and Science in Sports and Exercise, 25, 401-406. 
Haskell, W. I., Lee, I-M., Pate, R. R., Powell, K. E., Blair, S. N., Franklin B. A., Macera, C. A., Heath, C. W., Paul D. Thompson MD, and Adrian Bauman, A. (2007). Physical activity and public health: Updated recommendations for adults from the American College of Sports Medicine and the American Heart Association. Medicine and Science in Sports and Exercise, 39(8), 1423-1434.

Jackson, A. S., Blair, S. N., Mahar, M. T., Wier, L. T., Rossand, R. M., \& Stuteville, J. E. (1990). Prediction of functional aerobic capacity without exercise testing. Medicine and Science in Sports and Exercise, 22(6), 863-870.

Jackson, A. S., Dishman, R. K., La Croix, S., Patton, R., \& Weinberg, R. (1981). The heart rate, perceived exertion, and pace of the 1.5 mile run. Medicine and Science in Sports and Exercise, 13(4), 224-228.

Kasch, F. W. (1961). A comparison of the exercise tolerance of post-rheumatic and normal boys. Journal of the Association for Physical and Mental Rehabilitation, 15, 35-40.

Keen, E. N., \& Sloan, A. W. (1958). Observations on the Harvard step test. Journal of Applied Physiology, 13(2), 241-243.

Kohl, H. W., Blair S. N., Paffenbarger, Macera, \& Kronfeld. (1988). A mail survey of physical activity habits as related to measured physical fitness. American Journal of Epidemiology, 127(6), 1228-1239.

Kulberg, J. M., \& Kasch, F. W. (1979) Validation of the Kasch 3 minute step test. Medicine and Science in Sports and Exercise, 11(1), 94.

Leger, L. A., Mercier, D., Gadoury, C., \& Lambert, J. (1988) The multistage 20 meter shuttle run test for aerobic fitness. Journal of Sports Sciences, 6, 93-101. 
McArdle, W. D., Katch, F. I., Pechard, G. S., Jacobson, L., \& Ruck, S. (1972). Reliability and interrelationships between maximal oxygen intake, physical work capacity and step test scores in college women. Medicine and Science in Sports and Exercise, 4, 182-186.

Myers, J. (2003). Exercise and cardiovascular health. Circulation, 107, E2-E5.

Noble, B. J., Borg, G. A. V., \& Jacobs, I. (1983) A category-ratio perceived exertion scale: Relationship to blood and muscle lactates and heart rate. Medicine and Science in Sports and Exercise, 15(523-528).

Pate, R. R., Pratt, M., Blair, S. N., et al. (1995). Physical activity and public health: A recommendation from the Centers for Disease Control and Prevention and the American College of Sports Medicine. Journal of the American Medical Association, 272, 402407.

Petrella, R. J., Koval, J. J., Cunningham, D. A., \& Paterson, D. H. (2001). A self-paced step test to predict aerobic fitness in older adults in the primary care clinic. Journal of American Geriatrics Society, 49, 632-638.

Rhyming, I. (1954). A modified Harvard step test for the evaluation of physical fitness. Arbeitsphysiologie, 15, 235-250.

Ross, R. M., \& Jackson, A. S. (1990). Exercise Concepts, Calculations, and Computer Applications. Carmel, IN: Benchmark Press.

Santo, A. S., \& Golding, L. A. (2003). Predicting maximal oxygen uptake from a modified 3minute step test. Research Quarterly for Exercise and Sport, 74(1), 110-115.

Shahnawaz, H. (1978). Influence of limb length on a stepping exercise. Journal of Applied Physiology, 44, 346-349. 
Shephard, R. J. (1980). The current status of the Canadian home fitness test. British Journal of Sports Medicine, 14(2 \& 3), 114-125.

Siconolfi, S. F., Garber, C. E., Lasater, T. M., \& Carleton, R. A. (1985). A simple, valid step test for estimating maximal oxygen uptake in epidemiologic studies. American Journal of Epidemiology, 121(3), 382-390.

Tanaka, H., Monahan, K. D., \& Seals, D. R. (2001). Age-predicted maximal heart rate revisited. Journal of the American College of Cardiology, 37(1), 153-156.

U.S. Department of Health and Human Services. (2009a). Physical activity guidelines for Americans. Appendix 1. Retrieved from http://www.health.gov/PAGuidelines/guidelines/appendix1.aspx

U.S. Department of Health and Human Services. (2009b). Physical activity guidelines for Americans. Ch 8. Retrieved from http://www.health.gov/PAGuidelines/guidelines/chapter8.aspx

Watkins, J. (1984). Step tests of cardiorespiratory fitness suitable for mass testing. British Journal of Sports Medicine, 18(2), 84-89.

Watkins, J., \& Ewing, B. (1984). The effects of practice and method of scoring on performance in a step test suitable for use in schools. Scottish Journal of Physical Education 12, 12 17. 
Appendix A

\section{Perceived Functional Ability}

Suppose you were going to exercise continuously on an indoor track for 1 mile. Which exercise pace is just right for you--not too easy and not too hard? Circle ONE appropriate number (any number, 1 to 13 ).

1 Walking at a slow pace (18 minutes per mile or more)

2

3 Walking at a medium pace (16 minutes per mile)

4

5 Walking at a fast pace (14 minutes per mile)

6

7 Jogging at a slow pace (12 minutes per mile)

8

9 Jogging at a medium pace (10 minutes per mile)

10

11 Jogging at a fast pace (8 minutes per mile)

12

13 Running at a fast pace (7 minutes per mile or less)

How fast could you cover a distance of 3-miles and NOT become breathless or overly fatigued? Be realistic. Circle ONE appropriate number (any number, 1 to 13).

1 I could walk the entire distance at a slow pace (18 minutes per mile or more) 2

3 I could walk the entire distance at a medium pace (16 minutes per mile) 4

5 I could walk the entire distance at a fast pace (14 minutes per mile) 6

7 I could jog the entire distance at a slow pace (12 minutes per mile) 8

9 I could jog the entire distance at a medium pace (10 minutes per mile) 10

11 I could jog the entire distance at a fast pace ( 8 minutes per mile) 12

13 I could run the entire distance at a fast pace ( 7 minutes per mile or less) 
Appendix B

\section{Physical Activity Rating}

Circle the ONE number that best describes your overall level of physical activity for the previous 6 MONTHS:

$0=$ Avoid walking or exertion; e.g., always use elevator, drive when possible instead of walking

1 = Light activity: walk for pleasure, routinely use stairs, occasionally exercise sufficiently to cause heavy breathing or perspiration

2 = Moderate activity: 10 to 60 minutes per week of moderate activity; such as golf, horseback riding, calisthenics, table tennis, bowling, weight lifting, yard work, cleaning house, walking for exercise

3 = Moderate activity: over 1 hour per week of moderate activity as described above

4 = Vigorous activity: run less than 1 mile per week or spend less than 30 minutes per week in comparable activity such as running or jogging, lap swimming, cycling, rowing, aerobics, skipping rope, running in place, or engaging in vigorous aerobic-type activity such as soccer, basketball, tennis, racquetball, or handball

5 = Vigorous activity: run 1 mile to less than 5 miles per week or spend 30 minutes to less than 60 minutes per week in comparable physical activity as described above (\#4)

6 = Vigorous activity: run 5 miles to less than 10 miles per week or spend 1 hour to less than 3 hours per week in comparable physical activity as described above (\#4)

7 = Vigorous activity: run 10 miles to less than 15 miles per week or spend 3 hours to less than 6 hours per week in comparable physical activity as described above (\#4)

8 = Vigorous activity: run 15 miles to less than 20 miles per week or spend 6 hours to less than 7 hours per week in comparable physical activity as described above (\#4)

$9=$ Vigorous activity: run 20 to 25 miles per week or spend 7 to 8 hours per week in comparable physical activity as described above (\#4)

10 = Vigorous activity: run over 25 miles per week or spend over 8 hours per week in comparable physical activity as described above (\#4) 


\section{Appendix C}

\section{Physical Activity Readiness Questionnaire (PAR-Q)}

Common sense is your best guide when you answer these questions. Please read the questions carefully and answer each one honestly:

Check YES or NO:

YES NO

1. Has your doctor ever said that you have a heart condition and that you should only do physical activity recommended by a doctor?

2. Do you feel pain in your chest when you do physical activity?

3. In the past month, have you had chest pain when you were not doing physical activity?

4. Do you lose your balance because of dizziness or do you ever lose consciousness?

5. Do you have a bone or joint problem that could be made worse by a change in your physical activity?

6 . Is your doctor currently prescribing drugs for your blood pressure or heart condition?

7. Do you know of any other reason why you should not do physical activity?

\section{Do you have either of the following?}

If you are not feeling well because of a temporary illness such as a cold or a fever - wait until you feel better.

If you are or may be pregnant - talk to your doctor before doing a maximal test.

Informed use of the PAR-Q: Adapted from ACSM's Health/Fitness Facility Standards and Guidelines, 1997 by American College of Sports Medicine 
Appendix D

\section{R P E 15 Point Scale}

- $6-20 \%$ effort

- 7 - 30\% effort - Very, very light (Rest)

-

- $8-40 \%$ effort

-

- 9 - 50\% effort - Very light - gentle walking

$\bullet$

- $10-55 \%$ effort

$\bullet$

- $11-60 \%$ effort - Fairly light

-

- $12-65 \%$ effort

-

- $13-70 \%$ effort - Somewhat hard - steady pace

$\bullet$

- $14-75 \%$ effort

- $15-80 \%$ effort - Hard

-

- $16-85 \%$ effort

-

- $17-90 \%$ effort - Very hard

-

- $18-95 \%$ effort

-

- 19 - 100\% effort - Very, very hard

$\bullet$

- 20 - Exhaustion 